\title{
RÉGINE DOUADY
}

\section{Un processus d'apprentissage du concept d'aire de surface plane}

Publications de l'Institut de recherche mathématiques de Rennes, 1987-1988, fascicule 5 «Didactique des mathématiques », , p. 1-50

<http://www.numdam.org/item?id=PSMIR_1987-1988__5_A3_0>

(C) Département de mathématiques et informatique, université de Rennes, 1987-1988, tous droits réservés.

L'accès aux archives de la série «Publications mathématiques et informatiques de Rennes » implique l'accord avec les conditions générales d'utilisation (http://www.numdam.org/conditions). Toute utilisation commerciale ou impression systématique est constitutive d'une infraction pénale. Toute copie ou impression de ce fichier doit contenir la présente mention de copyright.

\section{Numdam}

Article numérisé dans le cadre du programme Numérisation de documents anciens mathématiques http://www.numdam.org/ 


\section{UN PROCESSUS D'APPRENTISSAGE DU CONCEPT D'AIRE DE SURFACE PLANE (1)}

Exposé présenté le 18 novembre 1988

par

\section{Régine DOUADY}

Sous le titre 'Ingéniérie didactique à propos des mesures de surfaces planes à l'école élémentaire"

\section{Introduction}

L'objet de ce travail est l'étude de la construction de la notion d'aire chez des élèves du cours moyen ( $9-11$ ans).

L'aire est un moyen de rendre compte de la place occupée par une surface dans le plan. Par le choix d'une unité, on peut assócier un nombre à une vaste famille de surfaces du plan (contenant toutes celles qu'on rencontre à l'école élémentaire et au collège). Si l'on change d'unité, les nombres changent mais les nouvelles mesures sont proportionnelles aux anciennes. Cependant, contrairement à ce qui se passe pour les segments, deux surfaces connexes auxquelles on associe le même nombre ne sont pas nécessairement superposables.

L'analyse que nous faisons nous amène à distinguer trois pôles en jeu : le pôle géométrique avec les surfaces considérées comme parties du plan, le pôle "grandeur" avec les aires (mathématiquement, l'aire peut être considérée comme une classe d'équivalence de surfaces, elle n'est pas définie pour les élèves mais on peut donner du sens à l'expression "avoir même aire") et le pôle numérique avec les mesures.

L'objectif de l'enseignement que nous cherchons à mettre sur pied est bien d'associer un nombre au maximum de surfaces (en particulier tous les polygones et les disques) de façon à pouvoir faire des comparaisons et des calculs. Cependant, pour définir une application mesure entre surfaces et nombres avec suffisamment de sens pour les élèves, nous faisons l'hypothèse qu'il faut d'abord construire l'aire comme grandeur autonome en distinguant aire et surface aussi bien qu'aire et nombre.

Un autre de nos objectifs est de différencier aire et longueur, en particulier aire et périmètre, et ceci avant même avant d'avoir un moyen de mesurer les aires - le périmètre est en effet, pour les élèves, une autre "mesure" de la surface. Nous retardons l'identification entre aire et nombre avec l'hypothèse qu'une identification précoce entre grandeurs et nombres favorise l'amalgame des différentes grandeurs en jeu (ici aire et longueur).

Nous avons donc élaboré un processus d'apprentissage conforme à ces hypothèses didactiques. Nous l'avons réalisé dans deux classes (un CMI, un CM2). L'analyse des productions des élèves en classe, au cours d'entretiens et d'épreuves écrites a permis de dégager les acquis, les difficultés qui résistent et les difficultés non prévues qui nous amènent à faire de nouvelles

(1) Cet article a été publié dans "Cahier de didactique des Mathématiques" de l'L.R.E.M. de Paris VII n 37 de mai 1987 sous la signature de R. DOUADY et M.J. GLORIAN. 
hypothèses didactiaues et à modifier les séquences.

Dans la première partie, nous développons notre problématique et formulons les hypothèses didactiques. Dans une deuxième partie, nous présentons les choix didactiques retenus pour la construction des séquences et les conceptions des élèves relevées au cours et à la fin de l'apprentissage. La troisième partie est consacrée aux résultats et à la formulation de nouvelles hypothèses didactiques. La quatrième partie est un retour sur l'ingéniérie didactique permettant de baser le processus d'apprentissage sur le déroulement de plusieurs dialectiques mettant en jeu différents sens de laire. Certaines étaient prévues dans le processus de départ, d'autres sont proposées ou renforcées à la suite de l'expérimentation.

\section{IProblématique}

La première question qui se pose esi de définir l'objet d'enseignement "aire" et pour cela de commencer par examiner la définition de l'aire dun point de vue mathématique de façon à adopter pour l'enseignement une présentation qui soit condée mathématiquement. Nous donnerons ensuite un aperçu du point de vue généralement adopté dans lenseignement et des difficultés observées chez les élèves avant de formuler nos hypothèses didactiques.

\section{Point de vue mathématique}

Dans toute la suite nous entendons par surface une partie du plan. La notion d'aire est un moyen de rendre compte de la place occupée par une surface dans le plan. L'objectif est de définir une application mesure d'un ensemble \& de surfaces planes à valeurs dans $\mathbf{R}^{+}$. Nous ne chercherons pas à caractériser les surfaces qui sont dans l'ensemble \&. L'important est pour nous qu'il contienne toutes les surfaces qu'on sera amené à mesurer à l'école élementaire et au collège. On peut dire que lensemble $s$ est contenu dans l'ensemble des parties de $\mathbb{R}^{2}$ intégrables au sens de l'intégrale de Riemann et qu'il contient toutes les parties compactes de $\mathbb{R}^{2}$ à bord $\boldsymbol{C}_{1}$ par morceaux.

L'application $F$ de $\&$ dans $\mathbf{A}^{+}$devra vérifier les propriétés suivantes:

- si $S_{1}$ et $S_{2}$ sont des surfaces "quasidisjointes" (i.e. qui niont en commun que des points du bord), alors $F\left(S_{1} \cup S_{2}\right)=F\left(S_{1}\right)+F\left(S_{2}\right)$ 
- sise sot $S$ d'intérieur non vide, alors $F(S)>0$

- pour toute isométrie $g$ de $\mathbf{R}^{2}$, et toute surface $S \in \&, F(g(S))=F(S)$.

On montre que pour tout $A \in \$$, d'intérieur non vide, il existe une et une seule application $F_{A^{\prime}}$ \& $\rightarrow-\rightarrow \mathbf{R}^{+}$vérifiant les propriétés ci-dessus et telle que $F_{A}(A)=1$.

A est la surface unitè pour la mesure (de surface) $F_{A}$ et $F_{A}(S)$ est la mesure de $S$ avec $A$ pour unitė. La mesure d'une surface est un nombre rẻel positif ou nul.

\section{Propriétés:}

- L'application $F_{A}$ est surjective : l'image de l'ensemble des carrés suffit à recouvrir $\mathbf{R}^{+}$mais elle n'est pas injective : à partir d'un rectangle, on peut toujours en fabriquer un autre non surperposable et de même mesure.

- L'application $F_{A}$ est croissante pour l'inclusion dans s et l'ordre usuel dans $\mathbf{R}^{+}$.

- Toutes les applications $F$ vérifiant les propriètés ci-dessus sont des $F_{A}$ pour un certain $A$.

- Toutes les mesures de surfaces définies comme ci-dessus sont proportionnelles:

si $B \in \delta$, posons $\lambda=F_{A}(B)$, alors $F_{A}^{\prime}=(1 / \lambda) F_{A}$ définie par

$\forall S F_{A}^{\prime}(S)=(1 / \lambda) F_{A}(S)$ est telle que $F_{A}^{\prime}(B)=1$.

L'unicité de $F_{B}$ entraîne $F_{B}=F_{A}^{\prime}=(1 / \lambda) F_{A}$.

- En particulier, si $B$ est telle que $F_{A}(B)=1$, alors $F_{A}=F_{B}$, si bien que pour une mesure $F$, on peut appeler unité n'importe quelle surface $S$ telle que $F(S)=1$.

\section{Aire d'une surface:}

Pour chaque choix de $A$, l'application $F_{A}$ permet de définir une relation d'équivalence $\boldsymbol{R}_{A}: S \boldsymbol{R}_{A} S^{\circ}$ si et seulement si $F_{A}(S)=F_{A}\left(S^{\prime}\right)$.

Les propriétés précédentes entraînent que les classes d'équivalence 
cotenues ne dependent pas du choix de $A$.

Nous appellerons aire d'une surface $S$ sa classe d'équivalence pour $n$ 'importe laquelle des relations $\boldsymbol{R}_{\boldsymbol{A}}$.

$F_{A}$ permet alors de définir une application $f_{A}$ de l'ensemble des aires sur $\mathbf{R}^{+}$de façon qu'on ait un diagramme commutatif :

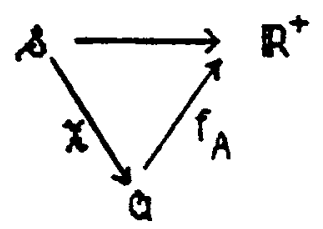

$x$ est lapplication qui, à toute surface associe sa classe d'équivalence. Si $S$ est une surface et $s$ sa classe d'équivalence, on a: $f_{A}(s)=F_{A}(S)$.

$f_{A}$ est bijective, cela permet de définir sur $Q$ une addition et un ordre tels que $f_{A}$ soit un isomorphime de $a$ sur $\mathbf{R}^{+}$.

On a alors:

- si $S_{1}$ et $S_{2}$ sont deux surfaces "quasidisjointes", $s_{1}$ l'aire de $S_{1}, s_{2}$ l'aire de $S_{2}$, s l'aire de $S=s_{1} \cup S_{2}$, alors $s=s_{1}+s_{2}$

- si $S_{1}=S_{2}$, alors $s_{1} s s_{2}$ où $s_{1}$ est l'aire de $S_{1}$ et $s_{2}$ l'aire de $S_{2}$

On peut aussi définir sur $\boldsymbol{Q}$ une multiplication externe de $\mathbf{R}^{+} \times \boldsymbol{Q}$ dans $\boldsymbol{Q}$ telle que si $\lambda \in \mathbb{R}^{+}$et $s \in Q$, alors $f_{A}(\lambda s)=\lambda f_{A}(s)$.

En fait, en mathématiques on garde rarement les trois termes surface, aire et nombre et le plus souvent on définit directement laire comme la mesure de la surface. Cette définition de l'aire la fait dépendre de lunité choisie, mais cela a peu d'importance puisque toutes les mesures sont proportionnelles, l'aire est alors dëfinie à un facteur multiplicatif près.

\section{Propriétés:}

- Déccupage-recollement : Soit une surface $S$ et une partition quelconque de $S$ en surfaces $S_{1}, S_{2}, \ldots S_{n}$ apparteriant a $B$. Considérons les surfaces $S_{i}=$ $g_{i}\left(S_{i}\right)$ ( $\mid$ sisn) où les $g_{i}$ sont des isométries quelconques du plan, alors la surface $S^{\prime}=U S_{i}$ a mème aire que $S$. En particulier deux surfaces superposables à découpage et recollement bord à bord près ont des aires 
égales.

- Dimensionalité de l'aire, bilinéarité de sa mesure par rapport aux mesures de longueur:

Choisissons un carré comme surface unité, la longueur $u$ d'un côté comme unité de longueur et son aire scomme unité d'aire. Un rectangle dont les côtés ont pour longueur $a u$ et bu a pour aire $a . b s$.

Si on applique au rectangle une homothétie de rapport $h$, les longueurs des côtés sont multipliées par $h$ et son aire par $h^{2}$. Plus généralement, on démontre par pavage, encadrement et passage à la limite que si on applique à une surface $S$ une homothétie (ou une similitude) de rapport $h$, son aire est multipliée par $h^{2}$. On retrouve cette propriété dans l'équation aux dimensions entre aires et longueurs.

\section{2) Point de vue généralèment adopté dans l'enseignement}

Nous avons vu que l'usage en mathèmatique est $d^{\prime}$ identifier $\boldsymbol{Q}$ et $\cdot \mathbf{R}^{+}$ grâce au choix d'une unité et de ne retenir que les deux pôles : surfaces et nombres. C'est le point de vue généralement adopté dans l'enseignement. Dans ces conditions, l'aire est un invariant non pas de la surface mais du couple (surface, unité) : pour une surface fixée, l'aire, considérée comme nombre dépend du choix de l'unité. C'est légitime quand on n'a pas l'intention de changer d'unité, mais c'est un point de vue difficile à tenir si on veut s'occuper de surfaces matérielles et si on veut que laire soit un invariant de la surface et d'elle seule.

La présentation standard consiste à recourir au pavage de surfaces, en passant rapidement au pavage avec des carrés et à compter les carreaux pour déterminer l'aire de surfaces quadrillées; ensuite, pour des rectangles ou carrés, à trouver des moyens économiques de comptage ; à introduire les unités légales avec multiples et sous multiples et selon le cas avant ou après cela, à établir les formules de calcul d'aire des rectangles en fonction des longueurs des côtés. On raccroche ensuite à ces formules celles relatives au triangle et à d'autres quadrilateres (losange, parallélogramme, trapèze).

Dans la majorité des manuels de 6e conformes aux programmes de 1977 (éditions de 1977 ou de 1981) l'aire est définie comme un nombre, parfois même comme un nombre de carreaux. Dans certains manuels, on n'envisage pas la possibilité de changer d'unité sinon de prendre des carrès de dimensions multiples ou sous multiples du carré unité. 
Les manuels qui ne veulent considérer que les deux pôles surfaces et nombres définissent laire comme un nombre mais utilisent l'expression "unité d'aire". Si l'aire est un nombre, que peut bien signifier une unité d'aire? On peut aussi lire "le $\mathrm{m}^{2}$ est laire d'un carré dont le côté mesure $1 \mathrm{~m}$ ". Si l'aire est un nombre, comment le $\mathrm{m}^{2}$ peut-il etre une aire ? Quand on arrive aux exercices tous les livres parlent d'une aire de $25 \mathrm{~m}^{2}$. IIs sont ainsi amenés à adopter, à un moment ou à un autre, sans le dire, le point de vue "grandeur", adopté explicitement par -d'autres manuels où le nombre est la mesure de l'aire.

Ainsi, même en admettant que l'objectif de l'enseignement est bien la construction et l'utilisation d'une fonction mesure qui associe un nombre à n'importe quelie surface, il semble bien qu'il y ait un troisième terme en jeu que l'on aura du mal à évacuer dans l'enseignement parce qu'il faudra bien un jour ou l'autre donner un statut à ces $25 \mathrm{~m}^{2}$ dont on aura besoin. Si on ne veut pas les identifier à la surface comme on le faisait autrefois, il va être aussi difficile de les identifier au nombre si on veut aussi que $25 \mathrm{~m}^{2}$ puisse être égal à $2500 \mathrm{dm}^{2}$ alors que $25=2500$. Ce troisième terme, c'est l'aire en tant que grandeur.

Ainsi qu'on vient de le voir, l'attention est essentiellement portée sur les deux pôles surfaces et nombres et ce d'un point de vue statique. On s'intéresse rarement à l'action des transformations, à la recherche d'invariants ou de modes de variations. Quand l'aire intervient, c'est très vite comme produit de longueurs avec des expressions numériques après choix d'unitès de mesure. 11 y a en général très peu de travail sur l'aire comme grandeur autonome.

\section{3) Quelgues difficultés et erreurs obsenvées chez les élèues}

* La surface unité étant une surface avec une certaine forme, la mesure d'une surface $S$ est tributaire de la possibilité de paver effectivement $S$ avec cette forme. Ainsi des èleves rencontrent des difficultés pour exorimer l'aire d'un triangle en $\mathrm{cm}^{2}$ puisquion ne peut pas le paver avec des carrés.

* L'aire est attachée à la surface et ne se dissocie pas d'autres caractéristiques de cette surface:

- si le périmètre d'une surface augmente, son aire aussi (et réciproquement)

- si deux surfaces ont le même périmètre, elles ont la même aire (et réciproquement). 
* On étend des formules à des situations où elles ne sont pas valables: par exemple produit des "dimensions" pour un parallélogramme ou des-"trois-... dimensions" d'un triangle.

* Lacquisition par les élèves de la "bidimensionalité" de l'aire (si les longueurs sont multipliées par $k$, l'aire est multipliee par $k^{2}$ ) pose problème. Cette question a èté ètudièe par J. Rogalski (1983).

\section{Hypothèses didactigues}

II nous semble qu'un certain nombre de difficultès sont liees au traitement par les èlèves des problèmes d'aire, soit du point de vue des surfaces, soit du point de vue des nombres.

Par exemple, une diminution de l'aire est comprise comme une diminution de la surface avec sa forme et va de pair avec une diminution du périmètre : l'aire et le périmètre sont alors amalgamés à la surface et liès à sa forme: on agrandit ou diminue la surface en conservant sa forme; le perimètre c'est le contour, l'aire c'est l'intérieur.

A l'autre extrēme, l'aire est un nombre : on est sur le plan du calcul et on ne relève que des èlements pertinents pour le calcul, par exemple des mesures de longueur qui paraissent caractéristiques de la surface considérée et qu'on combine dans des formules plus ou moins fondées : par exemple ajouter les mesures de deux côtés dun triangle et multiplier par la troisième, pour calculer l'aire du triangle en faisant le produit de deux longueurs.

Ainsi, au sujet de l'aire, les élèves développeraient une "conception forme" liee au cadre géométrique ou une "conception nombre" liée au cadre numérique, ou les deux, mais de façon indépendante l'une de l'autre, et ils traiteraient les problèmes sans établir de relation entre les deux points de vue. Or les problèmes d'aire mettent de façon essentielle en relation les cadres numérique et geométrique. Le concept d'aire en tant que grandeur constitue a notre avis un relais entre les surfaces et les nombres. Ceci nous amène à faire notre première hypothèse didactique :

(1) Le développement dans l'enseignement du concept d'aire en tant que grandeur permet aux élèves d'établir les relations nécessaires entre les deux cadres (gèométrique et numérique)

De plus, si lobjectif d'apprentissage est bien la construction et la manipulation d'une application mesure $F: s \longrightarrow \mathbb{R}^{+}$, il y a deux manières d'aborder la construction de cette application. 
Pour un choix fixe d'une surface unité $A$, par exemple un carré, il est facile d'associer un nombre à toute une catégorie de surfaces : celles pavables avec un nombre fini de copies de A. Le problème est d'enrichir l'ensemble des surfaces mesurables avec l'unité A. Par exemple quel nombre associer à un triangle ou à un disque ?

Pour une surface donnée 5 , deux cas se présentent:

1) on peut decouper $S$, ou un certain nombre d'exemplaires de $S$, en un nombre fini $k$ de pièces et recoller ces pièces sans chevauchement de manière à obtenir une surface $S^{\prime}$ pavable avec $A$.

Alors $F(S)=F\left(S^{\prime}\right)$, ou $k F(S)=F\left(S^{\prime}\right)$ selon le cas. On s'appuie sur l'additivité et l'invariance par déplacement.

2) on ne peut pas développer la procédure (1), on est alors conduit à une procédure d'encadrement de 5 par des surfaces pavables avec $A$ ou des subdivisions de $A$ et qui approximent 5 . de-mieux en mieux par f'intérieur et par l'extérieur.

Pour aborder la construction de l'application mesure, on peut soit utiliser les deux procédures soit niutiliser que la deuxième. En effet, par' la première procédure il est impossible d'atteindre toutes les surfaces de \&. La deuxième procédure est suffisante pour atteindre n'importe quelle surface de s et elle ne nécessite pas d'utilisation d'autre surface unité que des carrés. Cependant, nous pensons que la seule considération du deuxième point de vue ne permet pas à l'élève d'échapper à la prégnance de la forme des pièces et peut expliquer certaines des difficultés rencontrées. Nous faisons l'hypothèse que lutilisation du découpage-recollement permet l'élaboration du concept d'aire, étape-relais entre les surfaces et les nombres dans la construction de $F$, conformément à notre première hypothèse didactique.

D'autre part, si on prend comme unité l'aire d'un carré, on pourra éviter de dénombrer les unités en calculant sur les mesures. On aura alors besoin, pour construire $F$, d'établir des relations entre les mesures de longueur et les mesures d'aire.

Or la mesure permet d'identifier toutes les grandeurs (y compris longueurs et aires) a $\mathbb{R}^{+}$. Ceci est précieux du point de vue de la modélisation mathematique. Toutefois une identification trop précoce nous semble favoriser l'amalgame des différentes grandeurs alors que l'objectif à cet àge est plutôt de les différencier, et c'est sur quoi porte notre deuxième hypothèse.

(2) Une identification trop précoce entre grandeurs et nombres favorise l'amalgame des différentes grandeurs (ici. longueurs et 


\section{aires)}

Nous voulons eviter les confusions entre longueurs et aires, en particulier entre périmètre et aire, qui sont une des difficultés des élèves dans l'acquisition des mesures spatiales. Pour cela il nous parait important de développer des situations d'apprentissage dans lesquelles jouent de façon significative pour les unes les différences entre longueurs et aires, pour les autres les relations que ces grandeurs entretiennent. C'est pourquoi, dans notre ingénierie didactique, nous choisirons de dissocier les grandeurs (longueurs et aires) d'une part des objets-(segments, surfaces); d'autre part des nombres, avant d'aborder la mesure en fonction d'une unité choisie et les relations entre mesures de ces grandeurs.

\section{Il. Méthodologie}

\section{Ingéniérie didactique}

\section{a) Nos choix didactiques}

L'analyse précédente nous amène à distinguer deux points dans l'apprentissage :

a) construction de la notion d'aire comme grandeur autonome en faisant des comparaisons directes d'aires par inclusion, par découpage - recollement, et des mesures directes d'aire déduites du pavage à l'aide de pavés de formes varièes.

Cela nous amène à :

- dégager l'aire de la forme en différenciant aire et surface : deux surfaces de formes différentes peuvent avoir des aires égales.

- distinguer l'aire du nombre tout en contrôlant la correspondance : surfaces -- $\rightarrow$ nombres : à une même surface peuvent correspondre des nombres différents suivant l'unité choisie, mais l'aire, elle, ne change pas.

b) établir des relations entre aires et longueurs en s'intéressant à diverses transformations: les unes sont choisies pour pointer quaires et longueurs peuvent varier indépendamment l'une de l'autre, et même en sens inverse (aire et périmètre par exemple); les autres pour ètablir des relations 
entre aires et longueurs : calcul d'aires des surfaces usuelles, bidimensionalité. Ce faisant, on travaille à

- différencier aire et longueur en même temps qu'aire et surface

\section{b) Les séquences didactiques}

Nous avons contruit des séquences didactiques fondées sur les analyses précédentes et nous avons observé leur déroulement dans deux classes : un CM1 (9-10 ans) et un CM2 (10-11-ans). Les CM2 niavaient pas abordé la notion d'aire en CMI. On peut trouver dans (Douady - Perrin 84-85) ou (Douady Perrin 83) une description de la plupart des séquences didactiques utilisées.

Cependant ces documents ont été rédigés après le dérovlement complet en classe et l'observation des èlèves au cours d'entretiens par deux, ce qui fait-qu'on y trouve-des séquences qui niavaient pas été proposées aux élèves des deux classes concernées, mais qu'il nous a paru important d'ajouter.

En particulier, l'approche sur papier quadrillè n'a pas été réalisée parce que le comptage de carreaux nous semblait un acquis disponible des classes concernées. Cela s'est révélé exact. Cependạnt le recours au papier quadrillé est intéressant pour donner un premier sens au mot "aire"; sans rencontrer l'ambiguité qui reste attachée à des expressions comme "place occupée" qui pour les élèves font parfois davantage référence à l'encombrement (diamètre) qu'à l'aire. Dans la classe de CMI nous avions eu recours à la masse pour éviter ce problème. Nous considérons maintenant qu'un jeu de cadres entre papier blanc et papier quadrillé est un point important pour la mise en relation de différents sens de l'aire et l'ancrage de la dialectique surfaces aires - nombres (voir IV Retour sur l'ingéniérie didactique).

L'approche par les transformations n'a pas été proposée non plus aux élèves puisque son importance n'avait pas été prévue et que c'est un des résultats des observations et des entretiens individuels que nous avons eus avec les élèves après l'apprentissage.

Nous donnons ci-dessous la liste des séances réalisées dans chacune des deux classes. chaque seance de travail durait environ 1 heure $1 / 4$.

\section{Séquences réalisées en CMI}

* approche physique (1 séance: 9.12.1982)

Comparer des pièces en lino, en carton, selon la masse, selon la place occupée.

* approche géométrique (3 séances : $16.12 .82 ; 6.01 .83 ; 7.01 .83$ ) 
Découpages et recollements convenables à partir de rectangles : un mème rectangle pour les 4 membres d'une équipe, des rectangles différents pour deux équipes différentes. Découper les rectangles et recoller les morceaux sans en perdre et sans les faire chevaucher de façon que les quatre membres de l'équipe aient des surfaces de formes différentes. Décalquer les assemblages obtenus sur une feville blanche et comparer les nouvelles surfaces. Introduction du mot aire comparaison des aires des surfaces construites, au sein d'une équipe, au niveau de la classe.

* différenciation aire et périmetre (3 séances: 13.01.83; $27.01 .83 ; 3.02 .83$ )

- Commander de la ficelle pour border les surfaces fabriquées précé demment : deux surfaces de même aire peuvent avoir des périmètres différents.

- Modifier une surface donnée de façon à diminuer latre et:a augmenter le périmètre.

- Modifier un rectangle de façon à conserver l'aire et à augmenter. le périmètre.

* pavages et comparaison d'aires ( 3 séances : $3.03 .83 ; 4.03 .83$; 10.03.83)

Pavages de 4 surfaces à l'aide de pavés différents. Comparaison des aires des surfaces en ne disposant plus des surfaces elles-mêmes mais seulement des résultats du pavage et des pavés.

Les pavés ont été choisis de façon qu'il y ait des relations numériques entre eux faciles dans certains cas (rapports 2 ou 4), difficiles dans d'autres cas (rapports fractionnaires : $3 / 2,3 / 4$ ). Les surfaces étaient pavables par au moins un des pavés, ce qui donnait des comparaisons faciles entre certaines surfaces, difficiles entre d'autres et amenait à la nécessité de rechercher une unité commune ou de trouver au moins une des relations difficiles.

Surfaces proposèes :
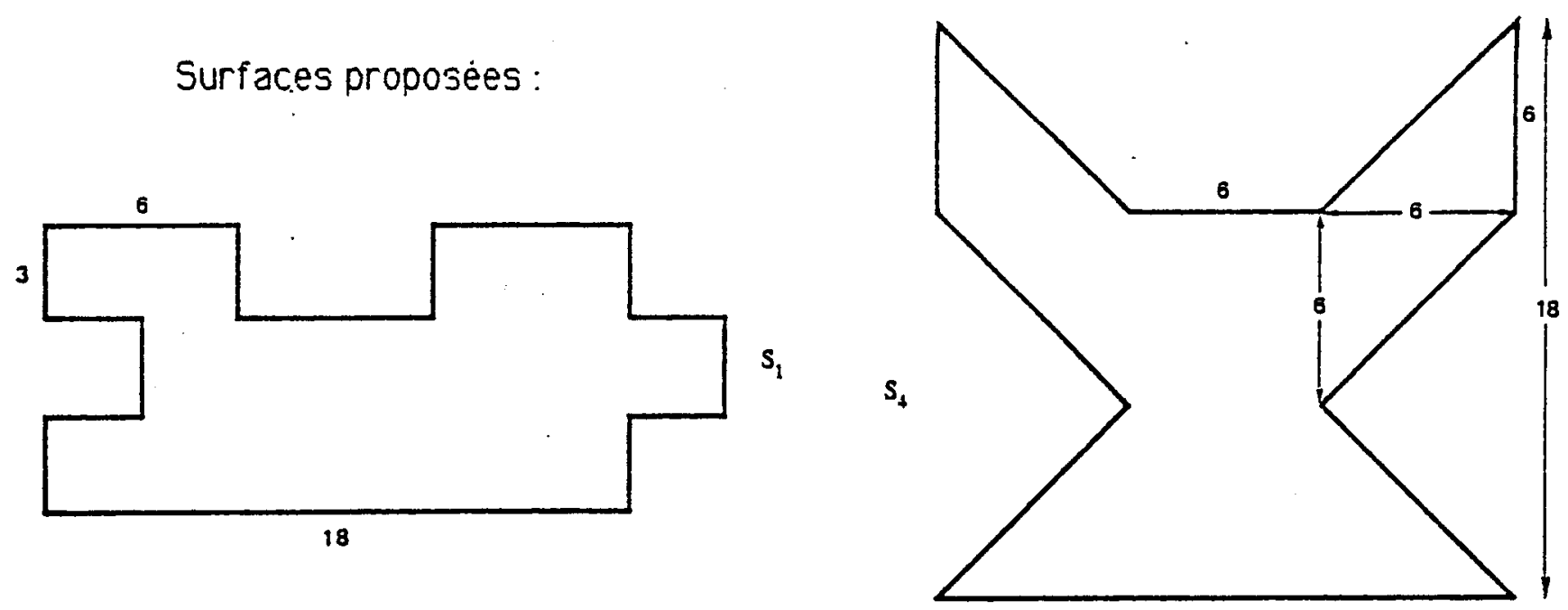


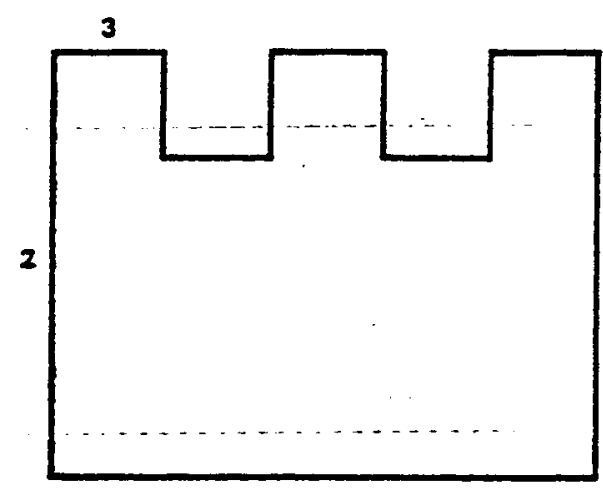

15

$S_{2}$

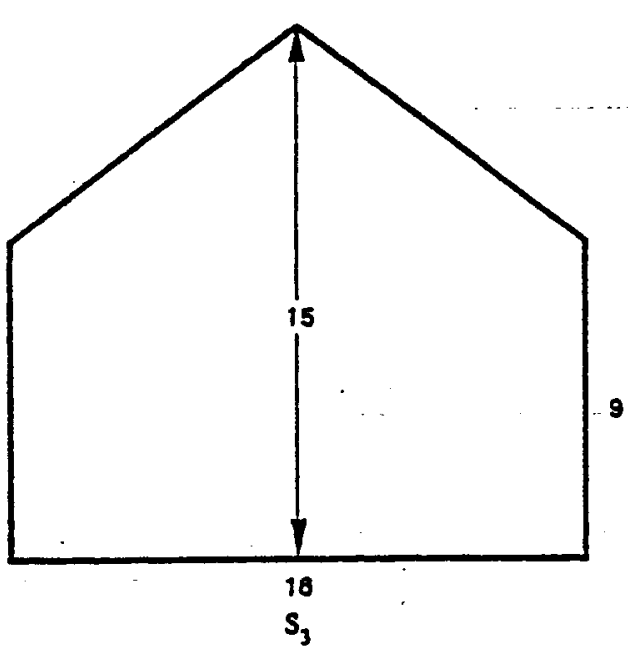
$S_{5}$ n'a été utilisé que par le CM2

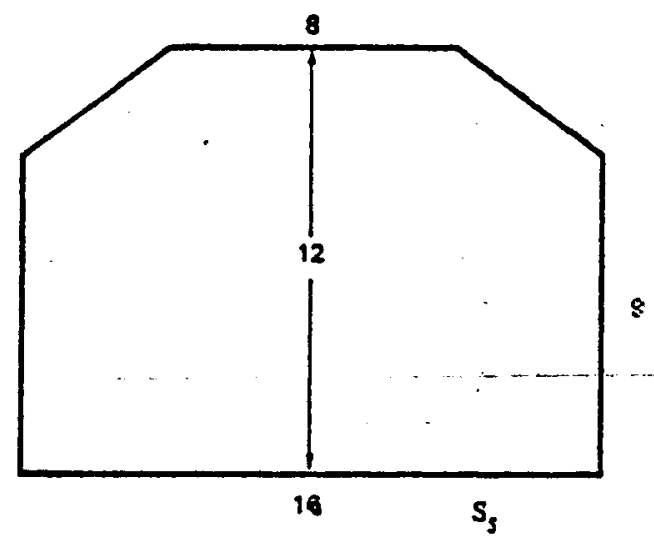

Pavés utilisés :

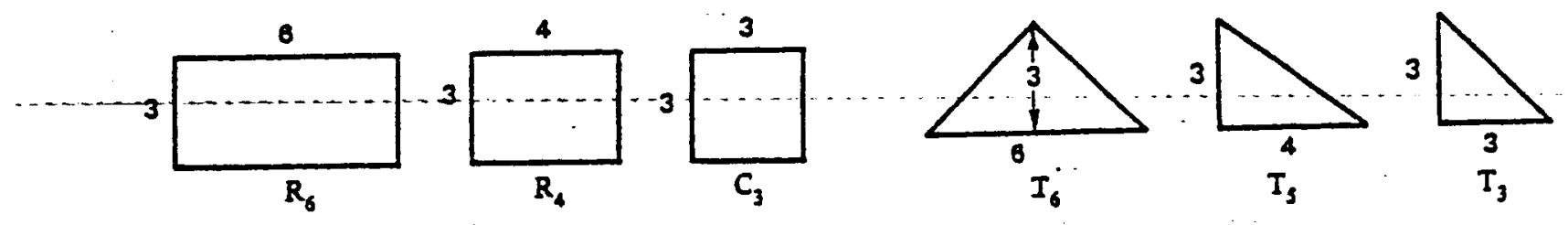

Séquences réalisées en CM2

* approche geométrique (3 séances : $10.01 .83 ; 14.01 .83$; 24.01.83)

les mêmes séquences qu'en $\mathrm{CMI}$

* différenciation aire et périmètre (une séance fin janvier 83 )

Commande de fil pour border les surfaces construites précédemment (cf $C M 1$ ) : deux surfaces de même aire peuvent avoir des périmètres différents

* pavages ( 3 séances : $17.02 .83 ; 24.02 .83 ; 26.02 .83$ )

Pavage de 5 surfaces à laide de pavés variés; recherche de relations entre les pavés. Les pavés étaient les mèmes qu'en CMI, il y avait une surface de plus. A cette étape les relations simples entre pavés sont trouvées et rassemblèes dans un tableau à double entrée, les résultats du pavage également. Le maitre introduit le mot "mesure".

* mesure et comparaison d'aires ( 4 séances : $28.02 .83 ; 4.03 .83$; $7.03 .83 ; 10.03 .83$ )

Les èlèves ne disposent plus des surfaces et doivent comparer les aires à l'aide des résultats du pavage. Cela a amené certains groupes d'èlèves à introduire de nouvelles unités, d'autres à tenter de compléter le tableau des relations entre carrelages.

On demande alors à tous les élèves de compléter ce tableau et 
d'exprimer les mesures de toutes les aires à l'aide de chacune des unités, y compris celles qui ont été introduites pour la comparaison....

Les élèves ont ensuite à mesurer une surface qui n'est pavable avec aucun des pavés considérés. On doit la couper en deux morceaux ou la remplacer par une surface de même aire et pavable.

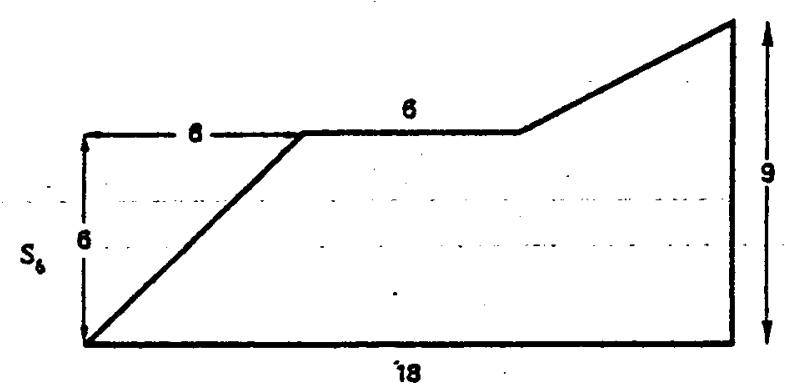

* Aires en $\mathrm{cm}^{2}$ (1 séance et demie : $15.03 .83 ; 17.03 .83$ )

Recherche de surfaces variées d'aire $1 \mathrm{~cm}^{2} ; 12 \mathrm{~cm}^{2}$

Recherche de rectangles daire $12 \mathrm{~cm}^{2}$.

* Encadrements: ( 1 séance et demie : 17.03 .83 et 18.03.83)

A l'alde de papier millimétré transparent, encadrements de l'aire d'une surface polygonale et dune surface à bords arrondis.
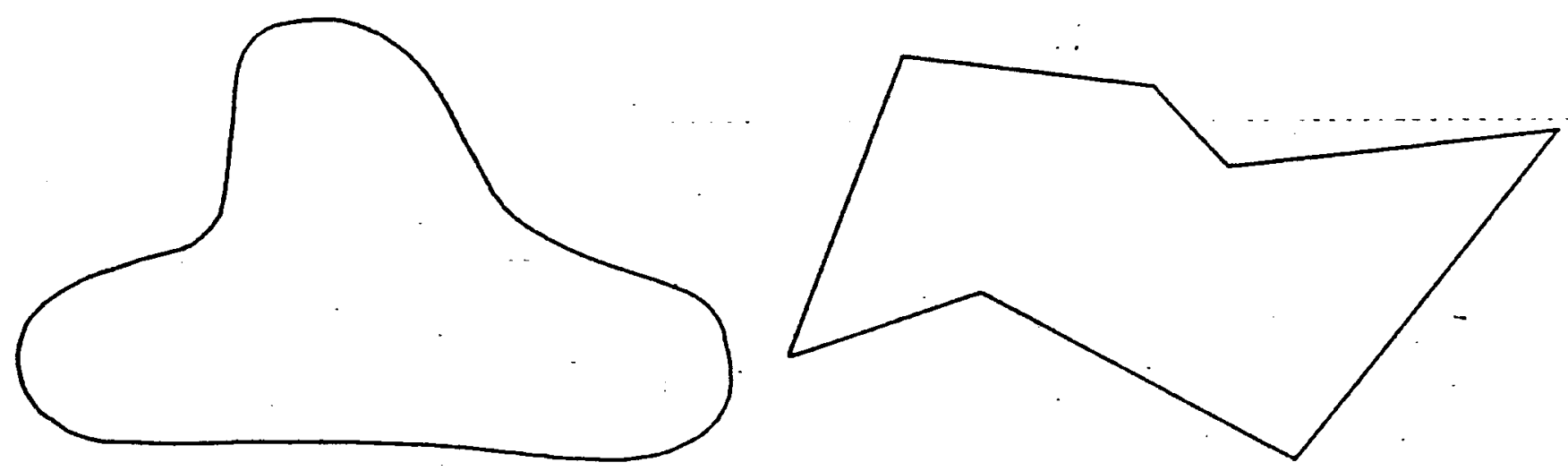

* Calcul diaires de rectangles ( 130583 ):

Recherche de rectangles à perimetre fixé, calcul de laire (l'interruption s'explique par les vacances de Pâques et un séjour en classe de nature).

\section{Obseruation des élèves}

Les séquences d'enseignement ont été observées. Nous avons pris des 
notes sur le déroulement. La plupart des productions des élèves ont été récupérées au CMI.

Les èlèves du CMI ont répondu à des épreuves écrites les 17/03/83 et 14/4/83.

Nous avons observé les élèves des deux classes au cours d'entretiens par deux, au CMI juste après l'apprentissage les 26 et 27 mars 1983, au CM2 deux mois après l'apprentissage entre le 20 et le 31 mai 1983.

Les élèves des deux classes ont passè en juin 1983 des tests écrits empruntés à J. Rogalski (1983).

\section{Etude des conceptions des élèves.}

\section{1) Au cours de l'apprentissage}

- Au démarrage, au CM2, certains enfants ont une conception de la place occupée par une surface liée à sa forme et -se référant plutôt à l'encombrement ou à la situation de la surface dans la feuille de papier. ou même à la manière dont elle a èté obtenue : pour comparer la place occupée par des surfaces obtenues par découpage et recollement sans perte $\mathrm{ni}$ chevauchement de rectangles superposables, des èlèves ne peuvent accepter qu'une surface encombrante (figure 1) puisse ne pas occuper plus de place qu'une autre plus "compacte" (figure 2)

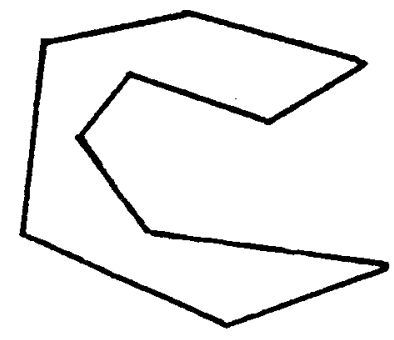

Fig 1

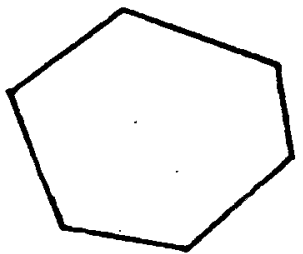

Fig 2

D'autres élèves veulent mesurer les distances des surfaces au bord de la feuille et d'autres ajouter les perimètres de tous les morceaux.

Mais ces difficultés ne résistent pas quand on parle de quantité de carton utilisée ou quand on fait référence à l'usage d'une balance:

A la séance suivante, les élèves du CM2 recourent tous à la comparaison des rectangles pour comparer toutes les surfaces de la classe du point de vue de l'aire. Le problème est plutôt de s'entendre sur ce que l'on veut comparer. L'introduction du mot aire dans un premier travail sur papier quadrillé évite cette ambiguité. 
En CMI, le recours à la masse a évité l'ambiguité de l'expression place occupée : si une surface se déduit d'une autre par découpage et recollement convenable, il est clair que la masse n'a pas changé (à la colle près) car la place des morceaux sur le plateau de la balance n'a pas d'importance et que les deux surfaces occupent autant de place car la référence est alors la quantitè de papier. Inversement, il n'est pas tout sûr pour les élèves que deux surfaces découpées dans le même carton et de même masse occupent autant de place : il faudrait trouver un découpage qui permette de reconstituer l'une à partir de l'autre.

\section{- L'amalgame entre aire et périmètre apparaît à plusieurs occasions :}

* quand il sagit de commander du fil pour border les surfaces en genëral très irrégulières fabriquées par les élèves à partir des rectangles : par exemple, un élève de CMI qui a une surface particulièrement compliquèe décide de se simplifier la tàche en mesurant le bord du rectangle témoin.

* en CMI, dans les épreuves écrites du 17 mars, lorsqu'il s'agit de dessiner un triangle de même aire qu'un rectangle donné, 13 élèves sur 24 fournissent un triangle de même périmètre que le rectangle.

* à la fin de l'apprentissage en CM2, cet amalgame entre conservation de l'aire et du périmètre réapparait lors d'une situation plus complexe : il sagit d'encadrer l'aire d'une surface a bords irréguliers et arrondis en utilisant du papier millimétré transparent; un (bon) élève de la classe demande de la ficelle pour se simplifier le travail en rectifiant le bord de la surface.

- La liaison entre surface et aire apparait quand on demande aux élèves de modifier une surface donnée pour en fabriquer une autre d'aire plus petite et de périmetre plus grand.

Pour diminuer laire, deux procédures sont utilisées : enlever un morceau, dessiner une surface à l'intérieur de la surface donnée.

11 semble que la première procèdure fasse davantage réfèrence à une conception de laire indépendante de la forme et la deuxieme a une conception liè à la forme. La consigne engage à une transformation: "modifier" ; la première procédure opère localement en enlevant un morceau ce qui change la forme de la surface ; la deuxième correspond à une vision plus globale de la surface qu'on essaie de modifier le moins possible en concevant une autre surface qui ressemble à la première mais située à 
l'intérieur de celle-ci : il semble que les élèves atttachent de l'importance à la forme de la surface et veuillent en modifier la taille sans en modifier la forme.

A ces deux procédures prenant en compte la variation d'aire à effectuer correspondent deux procédures pour prendre en compte la variation de périmètre :

* ceux qui enlèvent un morceau l'enlèvent toujours sur le bord (enlever un morceau à l'intérieur ne leur parait pas pertinent du point de vue du périmètre puisqu'ils ne considèrent que le périmètre extérieur) et ils s'aperçoivent assez vite que pour augmenter le périmètre, il faut remplacer un bord droit par un bord "en zigzag" ou courbe, même si leur premier essai diminuait le périmètre.

* les èlèves qui dessinent une surface à l'intérieur de la surface donnée font en général un bord assez parallèle au bord donnè Hs ont-diminué le périmètre. Pour rectifier, certains ont alors l'idée de "rajouter du périmètre" en doublant le bord de la surface intérieure, mais dautres restent bloqués.
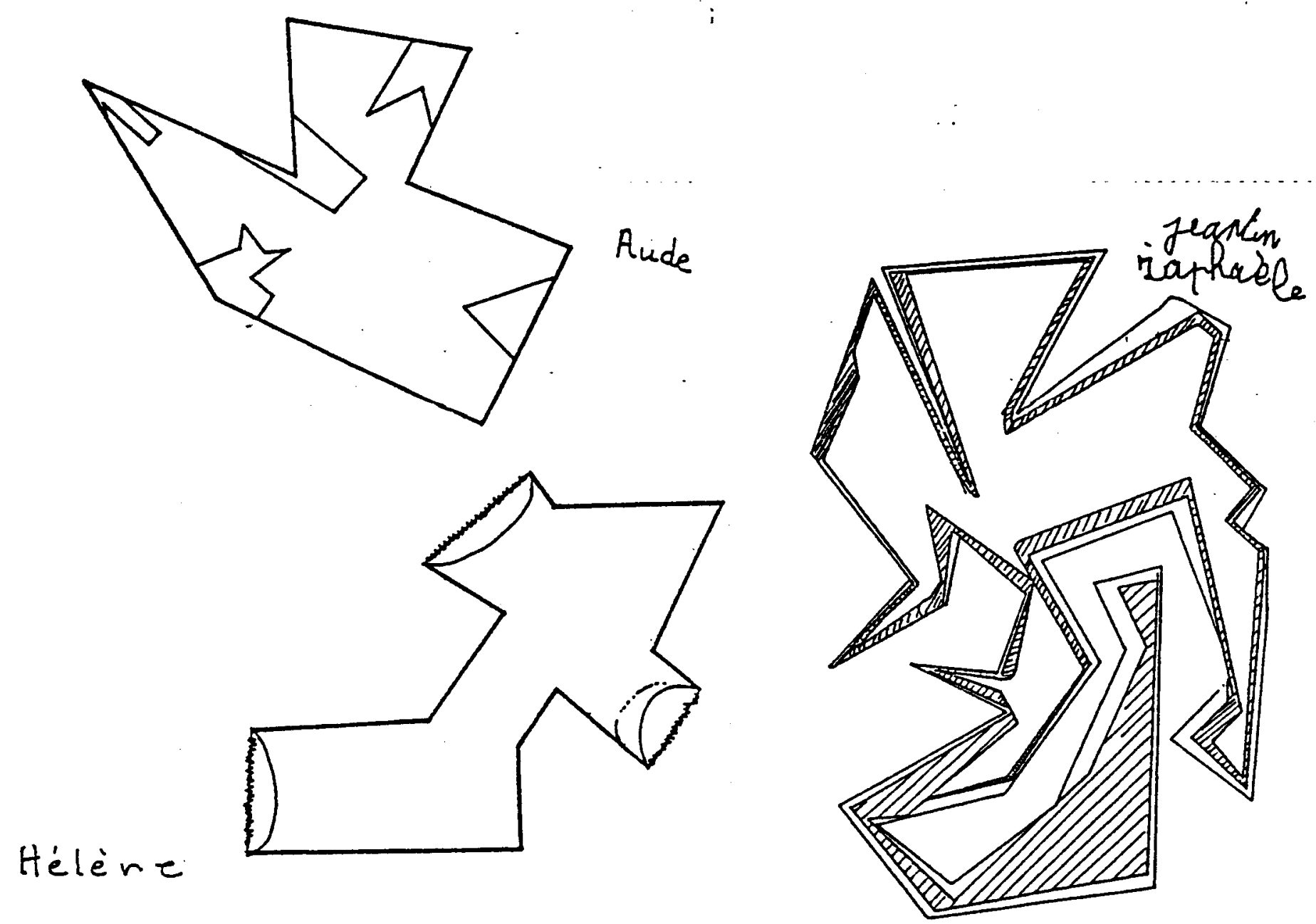

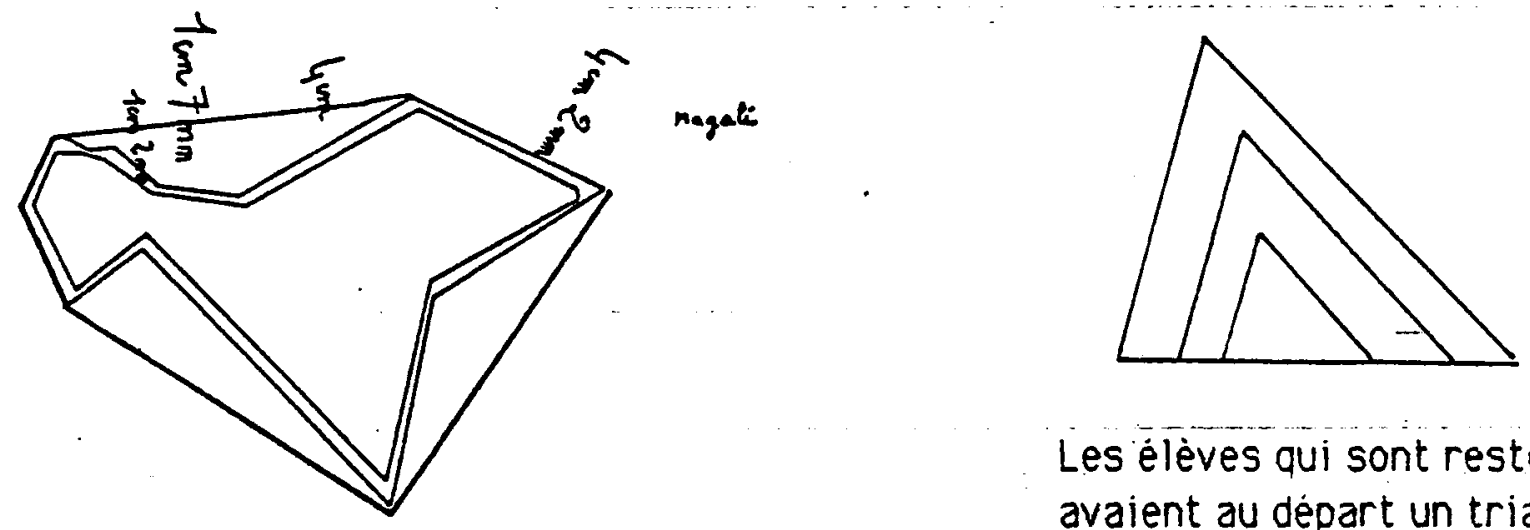

Les êlèves qui sont restès bloques avaient au départ un triangle et ont produit des triangles emboités

- approche de la mesure

Les élèves nont pas rencontré de difficulté pour associer des nombres différents à la même surface que ce soit pour exprimer le résultat de pavages avec des unitès différentes ou des mesures obtenues indirectement. Le recours aux nombres pour comparer des surfaces et la nécessitè d'utiliser une même unité pour mesurer les surfaces à comparer n'ont posé de problème pour personne. Les seules difficultés étaient súr les nombres dans le cas où ils étaient fractionnaires.

Au CMI, les èlèves n'ont pas produit d'unité nouvelle permettant de mesurer tous les pavés, ils niont pas non plus exprimé la mesure des pavés en fonction de chacun des autres : ils ont trouvé la relation $3 r=2 \mathrm{R}$ qui permettait de mesurer toutes les aires en prenant pour unité le rectangle d'aire $r$ par substitution de $3 r$ à $2 R$ et ils ont explicité quelques relations utilisant des $1 / 4$ ou des 1/3. Le travail fait auparavant sur les fractions n'étaient pas suffisant pour que tous les élèves expriment toutes les relations entre les pavés.

Au CM2, certains élèves ont recherché une unité commune permettant de mesurer les deux pavés rectangles : trois nouvelles unités ont été proposées, le rectangle $(1,3)$, le rectangle $(2,3)$ et le carré $(1,1)$. D'autres se sont lancés dans la recherche systematique de toutes les relations entre les unités d'aires utilisées, recherche qui sera ensuite effectuée par toute la classe de façon à complèter le tableau donnant les relations entre unités.

\section{2) Au cours des entretiens individuels}

Les entretiens individuels ont eu lieu au CMl deux semaines après l'apprentissage, au CM2 plus de 2 mois après l'apprentissage. Nous avons choisi d'interroger les élèves par deux pour observer ou le cas échéant 
provoquer des conflits de conception. Les élèves étaient choisis de façon qu'il n'y ait pas domination de l'un par l'autre.

Le problème était de compaier les aires des surfaces ci-dessous:

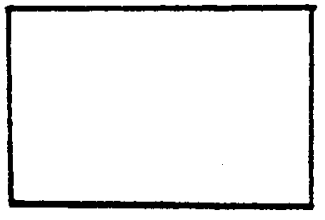

si

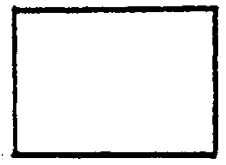

53

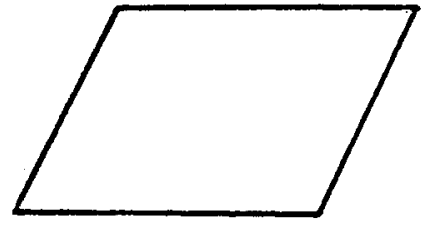

S2

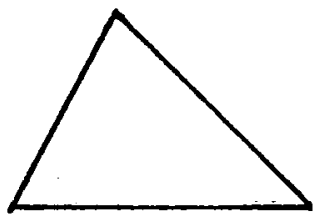

54

Les figures étaient dessinées sur papjer blanc, en cas de besoin, nous pouvions fournir les mêmes figures sur papier quadrillé au demi centimètre et aussi du papier blanc et du papier quadrillé au demi centimètre à volonté.

\section{Procédures observèes}

a) Ramener la comparaison des aires à la comparaison des nombres.

1) grâce au pavage : en comptant les carreaux sur papier quadrillé ou en quadrillant le papier blanc en carrés.

2) en faisant un produit de longueurs pour calculer les aires, en inventant au besoin des formules erronées (parallélogramme ou triangle).

Le désir de se ramener aux nombres est très fort, particulièrement au CM2 où on avait institutionnalise la formule de calcul de l'aire d'un rectangle et ou les èlèves travaillaient sur des calculs d'aires de rectangles pendant la même période en classe : beaucoup répétaient la question posee sous la forme "calculer les aires".

b) Ramener la comparaison des aires a la comparaison des longueurs des côtés (pour $S_{1}$ et 52 ).

c) Découper et recoller de façon convenable (pour comparer $S_{1}$ et $S_{2} ; S_{3}$ et $S_{4}$ ) ou paver $\left(S_{1}\right.$ avec $S_{3} ; S_{2}$ avec $\left.S_{4}\right)$. 
d) "Redresser" le parallélogramme pour en faire un rectangle ; "pencher" davantage le parallélogramme.

\section{Arquments utilisés par les élèves pour justifier leurs orocédures:}

a) Pour justifier un calcul:

- un parallélogramme a deux dimensions : pour calculer l'aire on multiplie les deux dimensions : $6 x(4+1 / 2)=27$.

Sur demande de l'interrogateur de montrer où sont les $27 \mathrm{~cm}^{2}$, certains èlèves justifient ce calcul en produisant un pavage par des petits parallélogrammes et déclarent : On a bien $27 \mathrm{~cm}^{2}$.

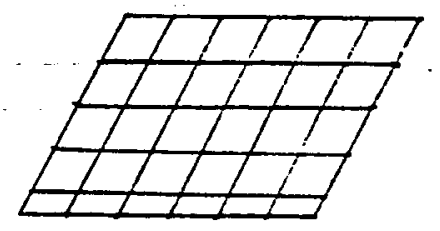

- pour le triangle

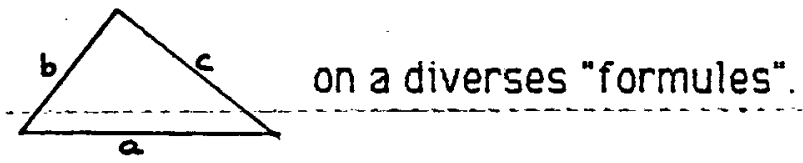

* une aire c'est un produit de 2 longueurs et on fait $(a+b) \times c$ pour le triangle (ou $a \times c+b \times c$ )

* un rectangle a deux dimensions, on multiplie pour trouver l'aire, un triangle a trois dimensions donc c'est $a \times b \times c$

* a $\times$ b $\times$ c donne un résultat beaucoup trop grand (par rapport a

51 et 53 ) et des élèves proposent alors $(a+b+c) \times 3$ ou même $a+b+c$. On assiste aussi au passage à des procédures périmétriques devant une difficultè de calcul: les cōtés obliques du triangle mesurent $4-1 / 2$ et $51 / 2$, un èlève veut multiplier les 3 côtès du triangle et ne sait comment multiplier $41 / 2$ par $51 / 2$; il décide alors de rassembler les $1 / 2$ et finit par ajouter les 3 dimensions.

b) Pour se ramener à la comparaison des longueurs :

$S_{1}$ et $S_{2}$ ont toutes les deux un côté de $6 \mathrm{~cm}$, on compare les autres côtés $4,5>4$ donc l'aire de $S_{2}$ est plus grande que l'aire de $S_{1}$.

c) Découpage-recollement: si on découpe un morceau d'une surface et qu'on le recolle sans chevauchement on conserve l'aire. Cet argument est correct et valorise dans lapprentissage ; mais les élèves qui ne peuvent utiliser que celui-là échouent dans la comparaison de $S_{3}$ et de $S_{4}$.

d) Déformation:

Si on "redresse" le parallélogramme, cela fait un rectangle de $4,5 \mathrm{~cm}$ sur $6 \mathrm{~cm}$ donc l'aire de $S_{2}$ est plus grande que l'aire de $S_{1}$.

\section{Conflits et changements de procédure}


II semble que les élèves aient plusieurs types de conviction qui les amènent à des conclusions opposées pour la comparaison de $\mathrm{S1}$ et de $\mathrm{S} 2$.

- Convictions permettant de conclure correctement $A_{1}=A_{2}$ :

* quand on découpe une surface et qu'on recolle convenablement les morceaux on a une surface de mème aire.

* pour comparer des surfaces dessinées sur papier quadrille on compare le nombre de carreaux qu'elles contiennent (éventuellement en regroupant des morceaux de carreaux pour faire des carreaux entiers). Au besoin on quadrille le papier pour faire apparaitre des $\mathrm{cm}^{2}$.

- Convictions amenant à la conclusion $A_{1}<A_{2}$ :

* quand on déforme un parallélogramme "en penchant plus ou moins" on conserve laire, en particulier un parallélogramme de $1 \mathrm{~cm}$ de côté a une aire de $1 \mathrm{~cm}^{2}$, mais aussi un parallélogramme obtenu à partir d'un carré de $1 \mathrm{~cm}$ de côté par découpage et recoilement: $1 \mathrm{~cm}$.

$\triangle$ a tous ses côtés qui mesurent

* l'aire du parallélogramme est le produit des dimensions, comme pour le rectangle.

Les deux types de convictions coexistent souvent chez le même élève. Pour certains groupes, on assiste mème à un va et vient des arguments contradictoires repris tour à tour par l'un ou l'autre des deux partenaires.

Les élèves ont différents moyens de résoudre la contradiction:

Le conflit se produit parfois spontanement par confrontation d'une procédure (c) et d'une procédure (d) et il arrive que les èlèves soient très étonnés de constater que le "côté droit" (hauteur du parallèlogramme) mesure $4 \mathrm{~cm}$ alors que le côté penché" mesure $4,5 \mathrm{~cm}$. Cela suffit parfois à résoudre la contradiction et,

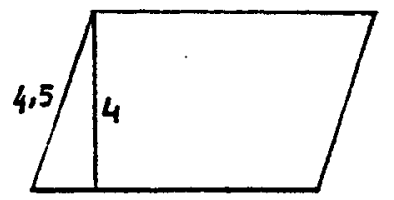
pour se convaincre, certains groupes dessinent sur la feuille d'autres côtés "de plus en plus penciés" et se convainquent de l'accroissement de la longueur de deux des cotés et de la conservation de laire du parallèlogramme. Inversement, cet accroissement de la longueur dans les cas extrèmes amène parfois les élèves à douter de la conservation de l'aire.

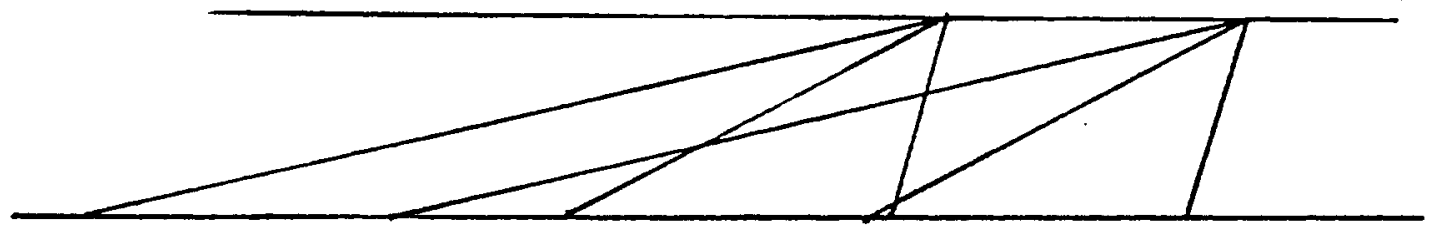


Pour d'autres groupes, le conflit se produit au moment où on leur fournit du papier quadrillé ou simplement au moment où on leur demande de dessiner les $\mathrm{cm}^{2}$ (après une réponse $A_{1}=24 \mathrm{~cm}^{2}, A_{2}=27 \mathrm{~cm}^{2}$ ). Le pavage du rectangle et du parallélogramme peut les amener à rassembler les deux pointes triangulaires du parallèlogramme pour obtenir des carreaux entiers et ainsi passer à une stratégie de type "découpage recollement".

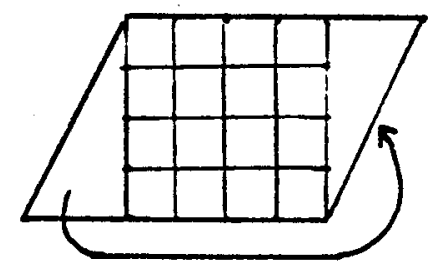

Pour dautres (procédure deformation), le changement de conviction s'opère souvent quand on leur propose de "pencher" de plus en plus le parallélogramme jusqu'à ce qu'il soit évident que l'aire est devenue très petite. 11 faut d'ailleurs noter la résistance des èlèves à pencher au-delà d'un certain seuil pour aboutir à des dessins comme celui-ci où on ne peut plus parler de "côté vertical":

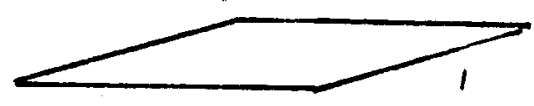

Pour ceux qui justifient le calcul avec un pavage de $S_{2}$ par des petits parallélogrammes de $1 \mathrm{~cm}$ de côté, le changement de conviction s'opère quand ils peuvent pointer la différence entre les 2 parallélogrammes:

- celui quion obtient à partir du carré de côté $1 \mathrm{~cm}$ a une aire de $1 \mathrm{~cm}^{2}$ mais les côtés ne mesurent pas tous $1 \mathrm{~cm}$ :

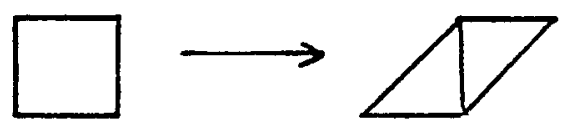

- celui quion obtient par déformation: tous ses côtés mesurent $1 \mathrm{~cm}$, mais son aire est plus petite que $1 \mathrm{~cm}^{2}$.

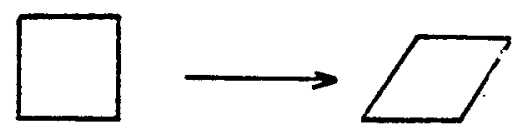

Notre interoretation est que beaucoup d'élèves amalgament trois transformations: le "parallelogramme articulé" qui conserve les longueurs des côtés mais non les aires, le glissement dun côté sur son suoport qui conserve les aires mais non les longueurs de tous les côtés et la rotation autour d'un sommet qui conserve à la fois les longueurs et les aires.

L'incidence sur la conservation des aires et des longueurs de 
transformations de figures par déformation continue est un point qui n'avait pas été abordé dans l'apprentissage et qui semble important pour déstabiliser des conceptions spontanées erronées des élèves à ce sujet et amorcer une autonomie des différentes notions amalgamées.

\section{3) Evolution des élèves du CM1}

Nous présentons ci-dessous l'évolution des élèves à travers l'épreuve écrite du 17/03, les entretiens en binômes des 26 et 27/03, l'épreuve écrite du 14/04 et des tests écrits en juin (cf ci-dessous 111 ). Nous interprétons les résultats à partir d'un tableau récapitulatif.

\section{a) Epreuve du 17/3}

texte:

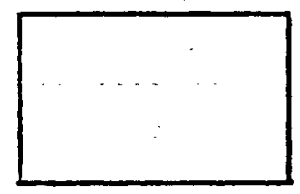

$S_{1} A_{1}$ est l'aire de $S_{1}$

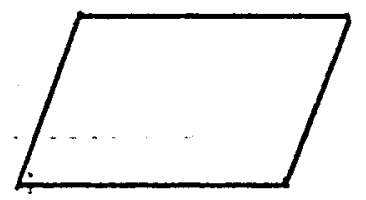

$\mathrm{S}_{2} \quad \mathrm{~A}_{2}$ est l'aire de $\mathrm{S}_{2}$

1) Comparer les aires $A_{1}$ et $A_{2}$

2) Dessiner un triangle d'aire $A_{1}$

3) Dessiner un rectangle d'aire $A_{2}$

\section{Les réponses}

Nous classons les procédures selon les outils mobilisés:

$(L)$ longueur ces èlèves comparent les longueurs des côtés de $S_{1}$ et $S_{2}$ ou les périmètres.

(D) déformation en faisant pivoter les côtés autour des sommets ou en faisant glisser un côté sur son support le parallélogramme se redresse en rectangle.

(DR) découpage-recollement ces èlèves découpent à gauche du parallélogramme un triangle qu'ils recollent à droite bord a bord sans chevauchement.

lère question: (L) 11

(D) 2 avec $A_{1}=A_{2}$

(DR) 11 dont 9 correctes, 1 estimation $A_{1}=A_{2}$ 1 réponse $A_{1} \neq A_{2}$ 
2ème question: (L) 13 ils dessinent un triangle $(6,6,8=4+4$ ) ou $(8,8,4)$

(DR) 2

rien ou essais infructueux 9

Parmi les 9 réponses (DR) correctes à la lère question, les réponses à la 2ème question se répartissent ainsi : 1 produit 2 triangles d'aire $A_{1}, 1$ a une procédure (DR) qui naboutit pas , 3 ont une procedure (L), 4 ne font rien.

Les $2(D)$ de la lère question adoptent (L).

3ème question: * parmi les 12 réponses $A_{1}=A_{2}$ à la lère question

- 5 proposent le rectangle $S_{1}$, dont 2 se référent à la lère question,

et 3 reproduisent le découpage et recollement.

-3 "redressent" et proposent le rectangle $(4+1 / 2,6)$

-4 ne font-rien

\section{* parmi les 8 réponses $A_{2}>A_{1}$}

6 "redressent" comme les précédents, 1 fournit un rectangle de même périmètre $(4,6+1 / 2), 1$ ne fait rien.

\section{b) Entretiens}

22 èlèves sont interviewés, 20 en binômes et 2 individuellement.

Parmi les binômes, 4 présentent des convictions qui s'opposent, chacun développant ses arguments, 4 présentent des oppositions plus molles, l'un des partenaires oscillant entre les convictions issues de la référence aux longueurs ou au découpage-recollement. Dans les deux autres cas, les partenaires s'accordent dans un binôme pour se rëférer aux longueurs des côtés, dans l'autre au découpage-recollement. Au cours des entretiens, les procédures des individus évoluent comme suit:

Au debut, $10(\mathrm{~L}), 2(\mathrm{D})$ et $10(\mathrm{DR})$. Parmi les (L), 7 changent ou oscillent, 3 vers (D) et 4 vers (DR). Dans les cas ou les conflits ne sont pas assez forts pour être dépassés par l'argumentation, c'est la référence au nombre de carreaux déterminé grâce au papier quadrillé ou gràce à un quadrillage du papier blanc qui résout le problème. Dans chaque binôme où l'un des partenaires avait une conception affirmée (DR), il a fait évoluer lautre, quitte à recourir au quadrillage. Cette évolution s'est confirmée lors de l'épreuve écrite du 14/04.

c) Epreuve du 14/04

texte: 


\section{c) Epreuve du 14/04}

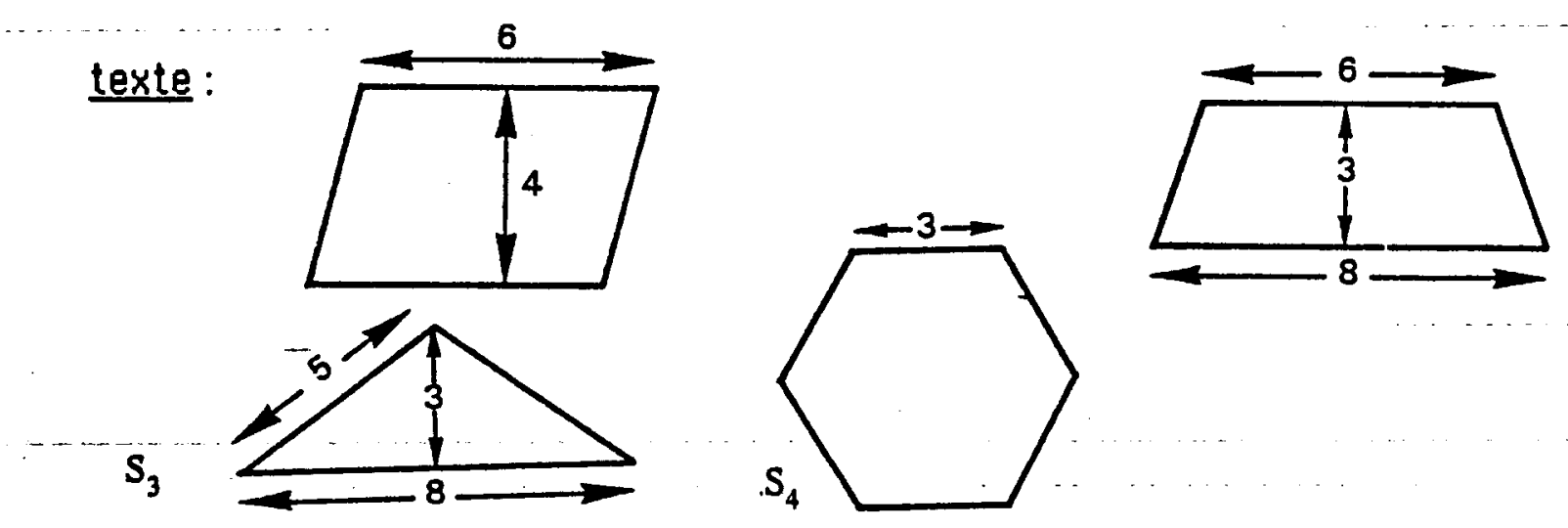

Pour cheque surface, dessiner un rectangle de mème sire.

\section{Les réponses}

Sur les. 21 réponses dépouillèes, une seule fait référence au périmètre pour dessiner un rectangle de même aire que l'hexagone.

9 produisent des rectangles corrects pour les 4 surfaces

8 produisent des rectangles corrects pour les 3 surfaces autres que l'hexagone: 6 n'en produisent aucune pour celui-ci, 1 essaie par découpage mais sans succès, 1 dessine une surface de mème aire non rectangulaire.

Les autres associent au parallèlogramme un rectangle de même aire et dessinent pour les autres surfaces des rectangles les encadrant ou contenus dedans.

\section{d) Tableau récapitulatif}

Nous présentons ci-dessous les conceptions des 18 élèves qui ont passé toutes les épreuves, telles qu'elles se sont manifestees aux trois occasions (17/03, entretiens $26-27 / 03,14 / 04)$ en ordonnant les élèves selon leurs performances en juin (voir textes en annexe) aux items. "combien de petites figures dans la grande" pour les carrés, parallélogrammes et triangles équilatéraux (rapports 2 et 3 ), "combien de pots de peinture pour peindre la grande surface" pour le triangle quelconque ( 8 items). Les 18 elèves ont répondu correctement aux questions concernant le carré et le paralle logramme agrandis dans les rapports 2 et $j$. Nous différencions les éleves selon leurs réponses dans les cas de triangles.

Dans le tableau ci-dessous, pour le 14/04, DR signifie que l'élève utilise le découpage-recoliement aux quatre questions avec réponses correctes. Dans les autres cas on indique le nombre de réponses utilisant correctement le découpage-recollement. Sauf précision contraire, il y a non-réponse dans les autres cas. Leilla a produit des rectangles ayant un lien géométrique avec la figure donnée mais pas la même aire, sauf dans un cas. 


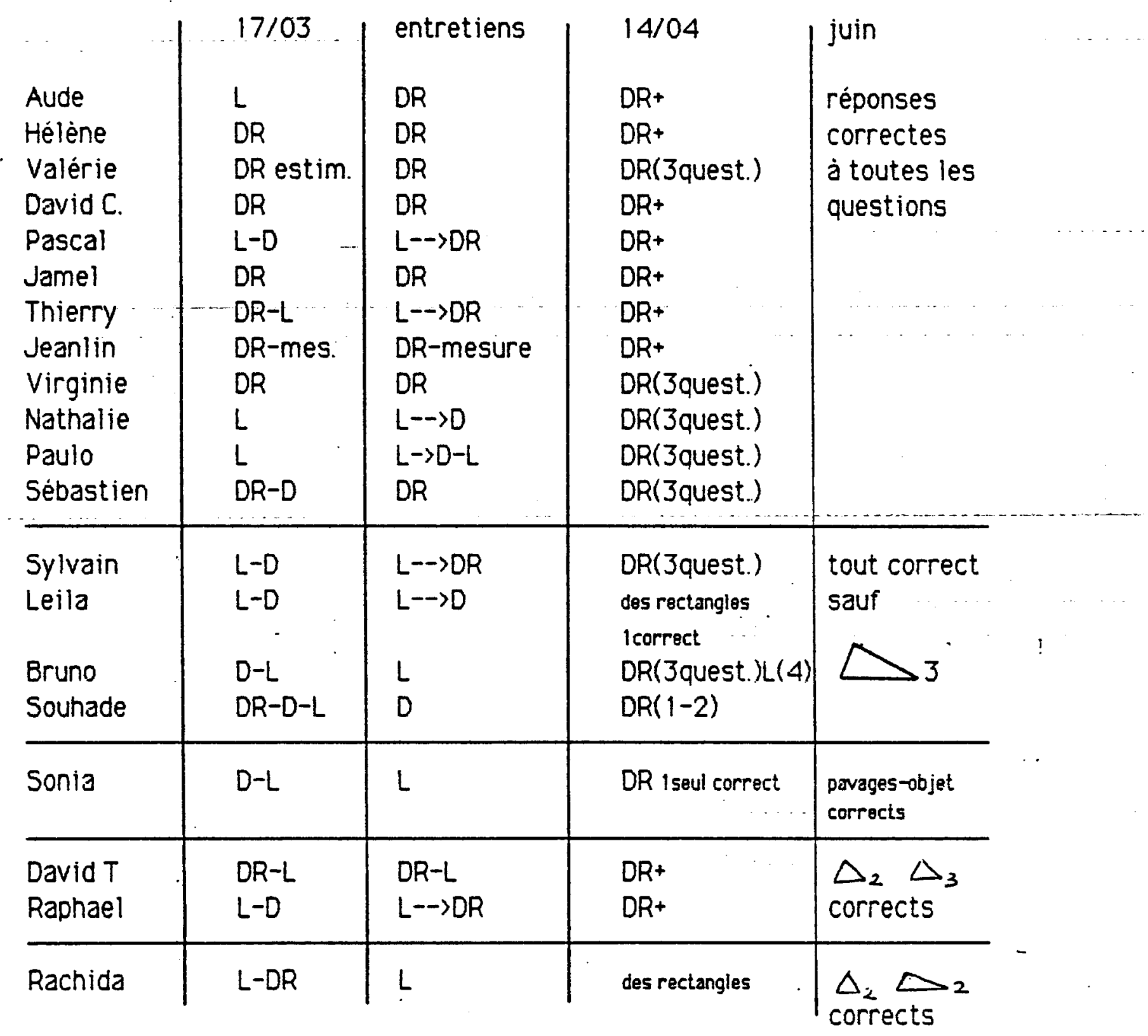

Conclusion: Une certaine dissociation aire-longueur s'est opérée pour la plupart des élèves. Les non-réponses le 14/04 l'emportent sur les réponses recourant au périmetre ou à la iongueur des côtés, en cas de difficulté. Nous reconnaissons là une évolution directement liée à l'apprentissage. Nous en testons la durabilite deux mois plus tard, grâce aux tests écrits de juin où le pavage est mobilisé comme outil. 


\section{Résultats}

\section{Les acauis}

Le pavage (en particulier le comptage de carreaux) est un outil disponible chez tous les éleves pour comparer des aires de surfaces planes de formes différentes. Nous l'avons constatè au cours des entretiens par deux et aussi dans les résultats obtenus aux tests écrits de fin d'année. En juin 1983; nous avons proposè aux élèves certains des questionnaires utilisès par J. Rogalski (Rogalski, 1983). Il sagit d'une variante du questionnaire SN, mais sans la phase 3 qui donne des exemples de pavage (textes en annexe).

\section{résultats à ces tests écrits:}

Nous avons retenu les réponses à trois questions:

a) Nombre de petites figures dans la grande.

Les figures utilisèes sont des carrés, parallelogrammes, triangles équilatéraux; les rapports sont 2 et 3 . Dans le tableau, nous donnons les résultats pour chaque figure avec en indice le rapport correspondant.

\begin{tabular}{l|c|c|c|c|c|c|}
\hline $\begin{array}{l}\text { Réponses } \\
\text { correctes }\end{array}$ & $\square_{2}$ & $\square_{2}$ & $\Delta_{2}$ & $\square_{3}$ & $\square_{3}$ & $\square_{3}$ \\
\hline $\mathrm{CM1}$ & 26 & 25 & 21 & 24 & 23 & 20 \\
\hline $\mathrm{CM}$ élèves & 26 & 21 & .18 & 22 & 18 & 17 \\
\hline 22 élèves & 22 & 21 & & & \\
\hline
\end{tabular}

Les nombres en italique dans les cases indiquent le nombre d'èlèves qui, parmi ceux de la case correspondante, ont donné une réponse correcte accompagnée d'un pavage par des surfaces de même aire que la petite surface donnée mais non superposables. Ainsi, deux élèves parmi les 21 qui ont donné une réponse correcte pour $\square$ ont dessiné

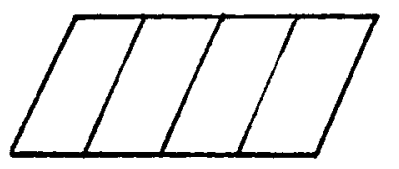

au lieu de

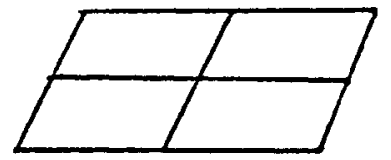

Remarquons que les parallélogrammes dessinés ont presque la même forme que le petit parallélogramme de départ. 
b) Nombre de Dots de peinture.

Les figures sont les mêmes que précédemment, avec en plus des triangles quelconques. On indique le nombre de pots de peinture nécessaires pour peindre la petite figure et on demande combien il en faut pour la grande.

\begin{tabular}{|c|c|c|c|c|c|c|c|c|}
\hline $\begin{array}{l}\text { Réponses } \\
\text { correctes }\end{array}$ & $\square_{2}$ & $\square_{3}$ & $\nabla_{2}$ & $\Delta 3$ & $\Delta 2$ & $\Delta 3$ & $\triangle 2$ & $\Delta 3$ \\
\hline $\begin{array}{l}\text { CMI } \\
26 \text { élèves }\end{array}$ & 18 & 14 & 16 & 14 & 19 & 15 & 16 & 14 \\
\hline $\begin{array}{l}C M 2 \\
22 \text { élèves }\end{array}$ & 19 & 21 & 18 & $19:$ & 22 & 21 & $16 z$ & 16 \\
\hline
\end{tabular}

c) Question en $\mathrm{cm}^{2}$ ?

La même question ètait posèe à propos de l'aire en $\mathrm{cm}^{2}$. Le carré ètait remplacé par un rectangle. Cette question n'a pas été posée aux éléves de CMI.

\begin{tabular}{l|c|c|c|c|c|c|c|}
\hline $\begin{array}{l}\text { Réponses } \\
\text { correctes }\end{array}$ & $\square_{2}$ & $\square 2$ & $\square_{3}$ & $\Delta 2$ & $\Delta 3$ & $\Delta_{2}$ & $\Delta_{3}$ \\
\hline $\mathrm{CM} 2$ & 20 & 21 & 19 & 18 & 18 & $15_{2}$ & $15_{4}$ \\
\hline 22 èleves & 20 & & & & & & \\
\hline
\end{tabular}

Exemples de pavages des triangles quelconques par des surfaces de même aire mais non superposables:
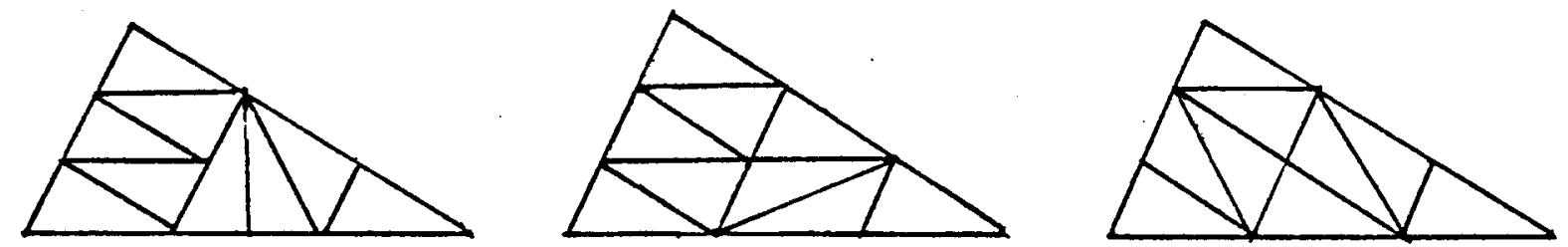

Les réponses que J. Rogalskki obtient à ces questions sont très différentes pour le carré (maitrisé dès le CMI) et pour le triangle : "il faut 
une longue évolution pour que les représentations de pavage du triangle soient mobilisables ( $f$ in de 5ème). C'est seulement en fin de tème que ces représentations sont disponibles pour les $3 / 4$ des élèves." Pour le pavage spontané (question 1), elle trouve $48 \%$ (resp. 39\%) de réponses correctes pour le triangle équilatéral avec le rapport 2 (resp. rapport 3), toutes classes confondues (de 9 à 14 ans). Nous avons à cette question des réponses comparables à celles des élèves de 4ème (13-14 ans); on peut également noter que les résultats corrects sont du même ordre qu'il s'agisse de carrés, de triangles ou de parallélogrammes, qu'il s'agisse du rapport 2 ou du rapport 3. Le "taux de passage" d'une réponse correcte au pavage à une réponse correcte à la peinture (réussite peinture + pavage / réussite pavage) varie de $85 \%$ à 100\% au CM2 et de $58 \%$ à $90 \%$ au CM1 : ces taux sont comparables à ceux obtenus par J. Rogaiski deux ans plus tard.

On peut penser que le travail fait dans les séquences didactiques a rendu disponible le pavage de surfaces variées, ce qui fait que les élèves sont capables, deux mois après l'apprentissage, de répondre à un certain nombre.de questions classiques concernant les aires.

On a pu constater au cours des entretiens et des épreuves du 14 avril que le découpage - recollement fonctionne pour comparer des surfaces, même s'il entre parfois en competition avec d'autres conceptions issues d'autres points de vue.

\section{Les difficultés qui résistent}

Les élèves ont fait certains progrès du point de vue de l'indépendance de l'aire par rapport à la forme, du point de vue de la différenciation aire-périmètre mais nous avons vu que les conceptions erronées peuvent réapparaitre jusqu'à la fin de nos séquences dans des situations plus complexes ou quand il s'agit de figures geométriques usuelles. II semble que dans ce dernier cas on ait davantage de points de vue qui entrent en compétition, ce qui arnène les élèves à produire des réponses erronées.

\section{Les difficultés non préuues}

Il est apparu au cours des entretiens, à la fin des séquences réalisèes, que le point de vue "déformation continue" intervient fortement dans les représentations et dans les décisions des élèves, surtout à propos de surfaces usuelles. Ainsi, un parallèlogramme est vu comme un rectangle déformé, les longueurs des côtès ne varient pas dans la transformation, l'aire ne varie pas non plus - qu'il s'agisse d'une articulation autour des sommets (longueurs des côtés conservées) ou d'un glissement d'un côté sur son support 
(aire conservée). D'ailleurs, n'est-ce pas ce point de vue qui faisait réclamer de la ficelle à un élève de CM2 pour "transformer en rectangle" une surface aux bords arrondis.

\section{Nouvelles hypothèses didactiques}

Les observations faites nous amènent à ajouter des hypothèses pour la construction de nouvelles séquences didactiques. Nous avions prévu du travail dans le cadre géométrique sans prendre en compte le point de vue dynamique de la déformation.

Nous faisons maintenant l'hypothèse qu'une interaction entre les points de vue statique et dynamique est nécessaire dans la conceptualisation de la grandeur aire et dans sa dissociation de la longueur.

Le découpage - recollement et la déformation amènent à des conclusions contradictoires. Le comptage de carreaux sur papier quadrillé emporte la conviction et permet de trancher.

Nous prévoyons maintenant de renforcer le jeu de cadres papier blanc papier quadrillé au début du processus d'apprentissage pour établir que, pour deux surfaces dessinées sur papier quadrillé, avoir des aires égales a le même sens, quion se réfère au comptage de carreaux, au déplacement ou au découpage - recollement et récolter dans les deux cadres des propriétés établies dans l'un des deux.

Un des objectifs est, en fin de processus, d'utiliser efficacement le quadrillage et ses raffinements successifs afin d'encadrer de plus en plus précisément une surface par des surfaces dont on sait mesurer les aires. Nous faisons l'hypothèse que trois points importants interviennent pour cela:

- la maitrise du passage du point de vue "papier blanc" au point de vue "papier quadrillé" et réciproquement

- la dialectique statique - dynamique

- la dissociation aire - longueur.

\section{Retour sur l'ingéniérie didactique}

L'analyse du déroulernent effectif des séquences nous amene à réorganiser l'ingénierie didactique. Nous allons proposer la recherche de problèmes qui donnent lieu au dèroulement de différentes dialectiques mobilisant différents sens de l'aire et dont l'enchainement devrait permettre l'élaboration du concept d'aire dans son double aspect outil et objet. 


\section{Jeu de cadres : papier blanc, papier quadrillé}

Le travail sur papier quadrillé a pour but de mobiliser une conception de l'aire mesurée par le nombre de carreaux : deux surfaces $S_{1}$ et $S_{2}$ ont la même aire quand elles sont constituees du même nombre de carreaux, qu'elles

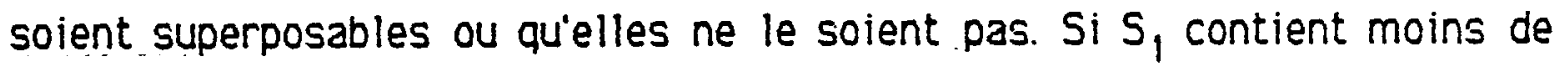
carreaux que $S_{2}$, l'aire de $S_{1}$ est plus petite que l'aire de $S_{2}$.

Le travail sur papier blanc a pour but de mobiliser une autre conception de l'aire, indẻpendante des nombres : deux surfaces $S_{1}$ et $S_{2}$ ont même aire

- si on peut trouver un déplacement (de l'espace) qui amène $S_{1}$ sur $S_{2}$; dans ce cas elles sont superposables

- si on peut découper l'une en un nombre fini de morceaux et les recoller sans chevauchement de façon à obtenir une surface superposable à l'autre.

Si un déplacement amène $S_{1}$ sur une partie de $S_{2}$, alors l'aire de $S_{1}$ est plus petite que laire de $S_{2}$.

11 est alors nécessaire de proposer une sitụation dont l'enjeu est de montrer que deux surfaces qui ont la même aire au sens du papier blanc l'ont aussi sur papier quadrillé :

- Etant donnée une surface $S$ dessinée sur papier blanc, les surfaces obtenues en reproduisant $S$ dans des positions quelconques sur un papier quadrillé contiennent le même nombre de carreaux (en particulier même si le bord ne suit pas partout les lignes du quadrillage)

- Etant donnée une surface $S$ dessinée sur papier quadrillé, toute surface obtenue à partir de 5 par découpage et recollement sans chevauchement contient le mème nombre de carreaux que $S$.

Ces deux critères nous garantissent la conservation du nombre de carreaux, qu'on connaisse ce nombre ou non. Ils fournissent les moyens de déterminer le nombre de carreaux contenus dans une surface en la remplaçant au besoin par une autre en contenant autant et pour laquelie le calcul est plus commode.

L'expression "avoir même aire" n'avait de sens au départ que pour des surfaces dessinées sur papier quadrillé et dont les bords suivent les lignes du quadrillage. Par un jeu de cadres papier blanc - papier quadrille, on etend ie champ des surfaces pour lesquelles lexpression "avoir même aire" a un sens.

\section{Remarque 1}

On ne peut faire l'économie du travail sur papier blanc. Pour les élèves privés des procédures sur papier blanc, les comparaisons d'aires de surfaces 
dessinées sur papier quadrillé conduisent à un comptage du nombre de carreaux en essayant de rassembler. des morceaux de façon parfois trés approximative, même dans le cas où les surfaces sont superposables. Le comptage peut ainsi amener des réponses contradictoires au sein de la classe. Les moyens de contrôle relèvent de procédures sur papier blanc.

\section{Remarque2}

Ce jeu de cadres papier blanc - papier quadrillé se situerait dans notre -progression au début de l'apprentissage, au CMI par exemple. On pourrait proposer les situations suivantes:

\section{Situations proposées}

- Tne prexiere situation a pour objectif d'introduire le mot "atre" en réterence au nombre de carreaux, de comparer les surfaces qui suivent les lignes du quadrillage (avec éventuellement des demi-carreaux). Deux surfaces ont la même aire si elles contiennent le nềe nombre de carreaux. One surface a une aire plus grande qu' une autre si elle contient plus de carreaux.

- La deurieze situation porte sur un des millons du jeu de cadres : $1^{\prime}$ inpariance du nombre de carreaux d'une surface lors d'um deplacement.

matériel : chaque élèe reçoit une feuille sur laquelle le mitre a dessine un rectangle et des segments arant pour longueur une des dimensions du rectangle, en diverses positions - suivant les lignes du quadrillage ou en oblique - 11 reçoit aussi du papier calque.

consigne : construire sur la feuille des rectangles dont 1 ' un des côtés est l'un des segments dessines et de mène aire que le rectangle donné. 1 chaque segment correspond un rectangle.

Le travail est indiriduel.

Bilan : In déplaçant un rectangle de $n$ 'importe quelle façon, par exemple en le taisant tounner autour d'un point ou en le taisant glisser le long d'ume droite, on obtient un rectangle superposable. In particulier, on peut affirmer que les dimensions et le nombre de carreaur à l'intérieur - donc l'aire - sont conserpés, sans apoir besoin de connaitre les raleurs numeriques.

- La troisieze situation est une reprise de la premiere apec des surfaces comportant des lignes obliques joignant un noeud du quadrillage a un autre. Les éleres sont amenes a poir des triangles rectangles commes des demi-rectsngles et à faire des deplacements de triangles rectangles pour faciliter le comptage de carreaux.

- La quatrieze sitnation a pour but de tormuler les conditions pour qu'un "découpage-recollement" conserre l'aire, c'est-a-dire le nombre de carreaux d'une surface dessiné sur papier quadrillé.

on donne aux éleves plusieurs exemplaires d'une surface dessinée sur papier quadrillé : on leur demande d'en garder wo intacte et de découper les deux autres en 5 ou 6 morceaur de formes simples en suivant les lignes du quadrillage, puis de recoller les morceaur de 
façon a fabriquer deux surfaces différentes de la surface donnée dont I'une ait une aire égale et l'autre une aire plus petite. on demande ensuite de reproduire les surfaces sur papier blanc. Le bilan permet. d'enoncer les conditions de recollement qui conservent le nombre de carreaux. On est assuré d'apoir conserpé le nombre de carreaur en se basant sur le tait qu' on $n^{\prime}$ a pas change les morceaux : si on $n^{\prime}$ a pas fait chevaucher en recollant. Ies deur surfaces sont formés des mênes norceaux. Le nombre de carreaur $n$ 'a pas change dans les norceaux, il n'a pas changé dans la surface totale.

Cinquière situation : Jusqu'a présent les élezes ont trapaillé sur papier quadrille. 1ls ront waintenant trapailler sur papier blanc. Par equipes, a partir de rectangles superposables qu' ils doirent découper en quelques norceaur simples. on leur demande de fabriquer des surfaces de formes differentes en recollant tous les morceaux sans cherauchement et de comparer les surfaces obtenues. On décide que les surfaces obtenues ont mème aire puisque le recollement périfie les conditions déterminées précédenment sur papier quadrillé.

Siriene situetion : Intin on propose aux elleves de decouper une surface-dessinée sur papier quadrillé et arec des lignes du découpage qui, cette fois, ne suivent pas le quadrillage; on demande aux élères de fabriquer arec les morceaux. d'autres surfaces comportant le mème nombre de carreaur. Le découpage-recollement est cette fois le mozen de répondre a la question : on n'a pas besoin de compter les carreaux de la nourelle surface, la réversibilite du découpage assure sa conserration. Rematquons que dans une classe ou nous arions propose ce travail arant la phase sur papier blanc, les elèves ont essayé de compter les carreaux des nourelles surfaces, en eraluant des morceaur de carreaux.

\section{Confrontation longueur - aire}

Le sens de l'aire dont on dispose permet-il de résoudre des problèmes impliquant à la fois aire et longueur, en particulier:

- deux surfaces qui ont même aire ont-elles mème périmètre ?

- deux surfaces qui ont mème périmètre ont-elles même aire ?

- peut-on modifier une surface de façon à diminuer laire et augmenter le périmètre ? diminuer le périmètre et augmenter laire ?

A ce moment de l'apprentissage, on sait mesurer les iongueurs, on ne sait pas mesurer les aires mais on dispose de orocédures qualitatives pour les comparer. Ces questions ont pour but de destabiliser une conception de l'aire liée à la forme, et de préoarer le recours pertinent aux nombres pour traiter les problèmes d'aire.

Elles avaient été prévues dans la première analyse ou nous avions envisagé les problèmes suivants:

1. commander de la ficelle pour border les surfaces de même aire fabriquées par équipe à partir de rectangles superposables; comparer ensuite les périmètres de ces surfaces.

2. à partir d'une surface donnée, en fabriquer une autre d'aire plus petite et de périmètre plus grand 
3. à partir d'un rectangle, en fabriquer un autre de mème aire et de périmètre plus grand (et éventuellement de même aire et de périmètre plus petit).

4. après l'introduction de la mesure des aires et des formules pour le rectangle, recherche de rectangles de périmètre donné et calcul de leur aire.

Les premières séquences permettent une différenciation aire-périmètre dans un contexte découpage-recollement avec des surfaces de forme "quelconque", c'est-à-dire en fait irrégulière. Nous avions aussi prévu de dissocier aire et périmètre à l'intérieur de la forme rectangle, par le découpage-recollement et par la mesure.

Nous avons vu que des difficultes subsistent (èpreuve du 17/3, entretiens), en particulier parce que, dans le cas de figures usuelles (rectangle, parallélogramme), les élèves recourent plus facilement à d'autres points de vue comme celui de la déformation continue.

Les séquences prévues initialement pour différencier àire èt longueür nous semblent devoir être conservées, mais elles seront complétées, et c'est l'objet du paragraphe suivant, par la prise en compte d'un point de vue dynamique.

\section{Dialectique statique - dynamique}

Nous avons constaté au cours des entretiens que, pour comparer des aires de surfaces planes, les èlèves recourent à des déformations continues des surfaces données et qu'ils ont tendance à amalgamer la conservation des aires et celle des longueurs des côtés dans de telles déformations. Nous proposons d'ètudier séparément l'effet des transformations qu'ils envisagent sur les aires et sur les longueurs (côtés et diagonales) des rectangles.

\section{a) Glissement d'un côté sur son support}

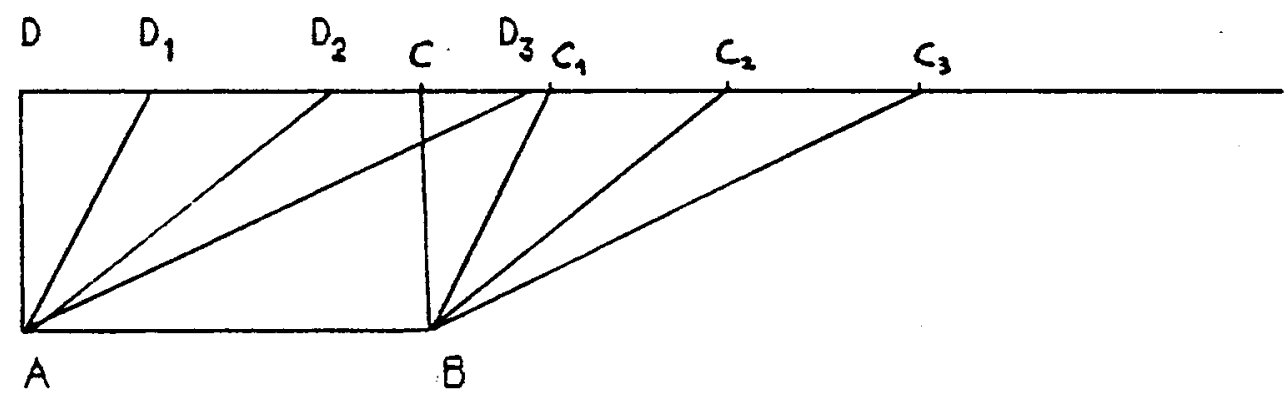

On déplace $C D$ sur son support (qui est la droite parallèle à $A B$ portant $C D$ ). Le rectangle $A B C D$ se transforme en parallélogramme. Comment varient les longueurs des côtés, de chaque diagonale, le périmètre, l'aire du parallélogramme? 


\section{b) Pivotement des côtès autour des sommets}

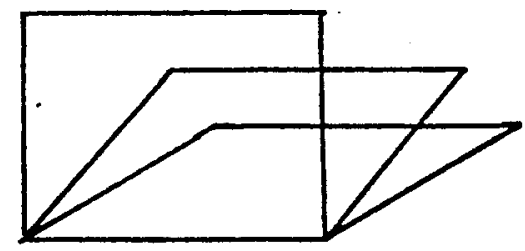

On a un parallélogramme articulé : les côtés sont des barres rigides (de longueur fixe) qu'on peut faire pivoter autour des sommets. Comment varient les longueurs des côtés, des diagonales, le périmètre, l'aire?

Les transformations continues $\mathrm{ci-dessus}$ se situent dans un cadre dynamique.

Le point de vue dynamique fait référence à des transformations continues et incite les élèves à considèrer oue les caractéristiques de la fiqure sont conservèes (en particulier aires et longueurs des côtès dans le cas a) cornme dans le cas b)) puisqu'il s'agit de la mêrne figure qu'on déforme : "on penche le rectangle", "on redresse le parallélogramme". Intéressons-nous aux élẹves pour lesquels un parallélogramme n'est: qu'un rectangle penché et qui proposent de "redresser" le parallélogramme pour obtenir un rectangle de même aire. Ces èlèves sont d'autant plus convaincus de leur démarche que le parallèlogramme se présente à eux comme un rectangle peu perturbé (côtés peu penchés). Les élèves s'écartent ainsi peu de la situation de départ, ce qui leur garantit une illusion de stabilité.

Pour dépasser ce point de vue, il est nécessaire de dissocier les états de départ et d'arrivée tout en les considérant comme deux ètats d'une déformation qu'on peut accuser vers des cas limites de façon à en grossir les effets : parallélogramme très allongé dans le cas a), très aplati dans le cas b).

La perception oblige à douter mais non à renoncer immédiatement aux convictions de départ.

Dans le cas b) en poussant la transformation, la perception à elle seule oblige à remettre en ouestion les convictions de depart puisqu'à la limite le parallélograrnme ạplati a une aire nulle. Cependant la variation de laire est perçue dans un premier temps de manière discontinue : d'abord elle ne chande pas, ensuite elle devient très petite. Puis, olusieurs étapes intermodiaires etant dessinées, on se convainc plus ou moins que laire diminue régulièrement, devient nulle à la limite. Remarquons que nous retrouvons là des observations analogues à celles de Vinh Bang et Lunzer (1965) au cours de l'experience suivante: un fil de longueur fixe est tendu entre les trois points $A, B, M$. $A B$ est fixe ; Mvarie d'une position centrale $M_{0}$ à une position limite $L$.

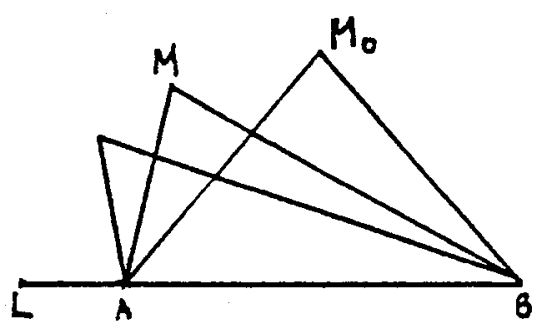


Les questions posées concernent la conservation de l'aire.

Dans le cas a), on constate que les côtés deviennent de plus en plus longs, mais du coup on se met à douter de la conservation de l'aire.

Des conjectures sont faites, des preuves deviennent nécessaires.

Dans le cas b), on peut recourir au découpage et au recollement pour avoir une preuve :

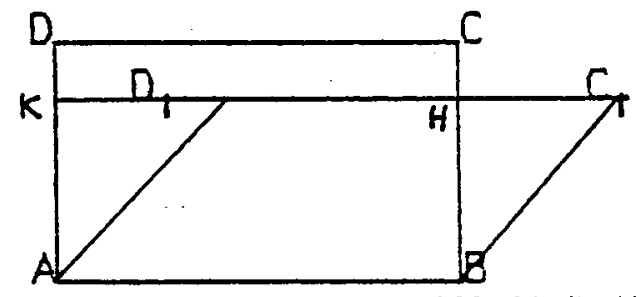

Le parallélogramme $A B C_{1} D_{1}$ a mème aire que le rectangle $A B H K$ et donc une aire plus petite que le rectangle $A B C D$. On remarque de plus que si les longueurs des côtés sont conservées, celless des diagonales, elles ne le s̀ont pas.

Dans le cas a) la perception peut faire douter de la conservation de l'aire; pour s'en convaincre il faut changer de point de vue et recourir à un découpage - recollement, du moins si CD ne s'est pas trop déplacé :

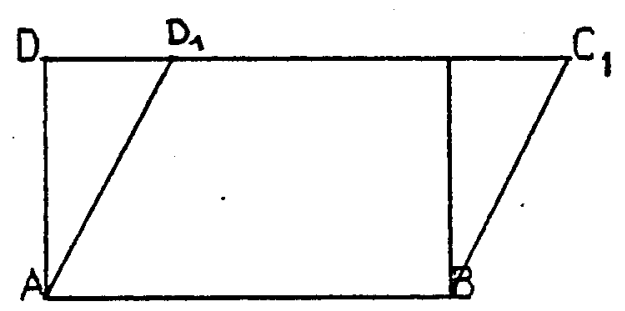

aire de $A D D_{1}=$ aire de $B C C_{1}$ donc aire de $A B C D=$ aire de $A B C_{1} D_{1}$

C'est bien un changement de point de vue puisque, si on peut voir le découpage - recollement comme une sorte de transformation, ce n'est pas une déformation continue : on ne regarde que l'état de depart et l'état d'arrivée. C'est en cela que le découpage - recollement est plus "statique".

Dans le cas oú $D_{1}$ est extérieur au segment $D C$, on a besoin de recourir à un point de vue tout à fait statique basé sur l'additivité des aires: 


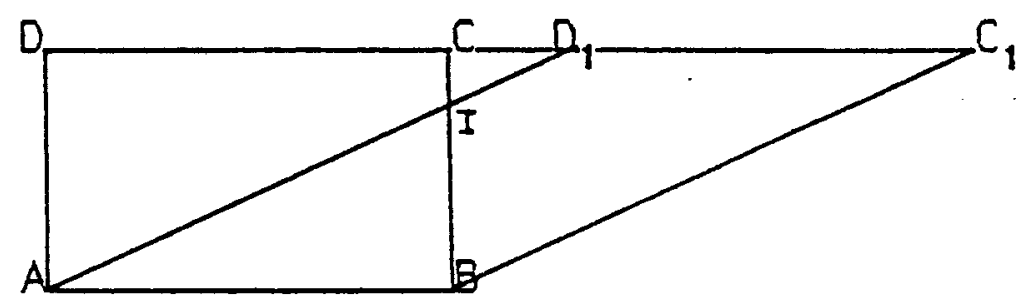

On a par exemple:

aire de $A B C_{1} D=$ aire de $A B C D$ + aire de $B C C_{1}$ = aire de $A B C_{1} D_{1}$ + aire de $A D D_{1}$ et aire de $A D D_{1}=$ aire de $B C C_{1}$ donc aire de $A B C_{1} D_{1}=$ aire de $A B C D$.

On pouvait aussi, en essayant de comparer directement $A B C D$ et $A B C, D$, voir d'une part que : aire de $A B C D=$ aire de $A B I+$ aire de $A I C D$ et aire de $A B C_{1} D_{1}=$ aire de $A B \mid+$ aire de $B \mid D_{1} C_{1}$,

d'autre part que aire de $A I C D=$ aire de $A D D_{1}$ - aire de $I C D_{1}$ et

aire de $B I D_{1} C_{1}=$ aire de $B C C_{1}$ - aire de $I C D_{1}$

or, aire de $A D D_{1}=$ aire de $B C C_{1} \ldots$

Le démarrage de la deuxième procédure peut paraitre plus facile puisquii correspond à l'habitude qu'ont les élèves de découper une surface en morceaux. Mais on est ensuite amené, comme dans le premier cas à insérer les surfaces qu'on veut comparer ( $A I C D$ et $B I C_{1} D_{1}$ ) dans des surfaces plus grandes $\left(A D D_{1}\right.$ et $\left.B C C_{1}\right)$, ce qui ne fait pas partie des habitudes. Dans ces conditions, le recours au trapèze est plus économique et on peut l'envisager même quand $D_{1}$ est entre $C$ et $D$.

De toute façon, on doit s'intéresser aux triangles $B C C_{1}$ et $A D D_{1}$.

Toutefois, pour se rendre compte de légalité de leurs aires, on a besoin de les considérer comme translatés lun de l'autre et donc d'avoir une vision dynamique de la figure mais en focalisant cette fois son intérêt sur les deux triangles et non plus sur la déformation du rectangle en parallélogramme.

Apparait alors un nouvel objet d'étuce qui est une autre procédure pour comparer: plonger les deux surfaces à comparer dans une troisième et se ramener a la comparaison des complements. Cette procedure est difficile pour les élèves de l'école èlémentaire et elle nécessite apprentissage. Notons que ce recours aux complements sera nécessaire dans dautres situations de mesure (par exemple comparaison de volumes pleins). La situation rencontrée ici offre une occasion de l'aborder.

Quant aux variations de longueur des côtés $A D$ et $B C$, si elles sont peu perceptibles quand $D_{1}$ est voisin de $D$, elles deviennent manifestes quand $D_{1}$ s'éloigne de $D$. 11 en est de même pour la diagonale $A C$. En revanche $B D$ diminue quand $D_{1}$ se rapproche de $C$, passe par un minimum quand $D_{1}$ est en $C$ et augmente ensuite. 
Le cadre dynamique devrait permettre de pointer les sources de difficultè, de faciliter les dissociations nécessaires: a) glissement, b) articulation.

Le cadre statique est le cadre adapté pour établir des preuves.

Ce faisant, le cadre géométrique s'enrichit d'un théorème : invariance de laire par "glissement". Ce théorème justifie la formule de l'aire du parallelogramme : aire $=$ base $\times$ hauteur.

Un nouveau problème s'impose pratiquement ici-taire dépend-elle de choix de la base? non d'après la formule usuelle, oui d'après les conditions de productions que nous avons proposees ( qu'il sagisse de découper et recoller un triangle ou de déformer le parallélogramme par glissement d'un côtè).

La réponse à ce problème amène au problème suivant:

On déforme un parallélogramme $A B C D$ en rectangle de deux manières:

$\begin{array}{ll}\text { 1) } A B \text { est fixe, } C D \text { glisse } & \text { 2) } A D \text { est fixe, } B C \text { glisse }\end{array}$

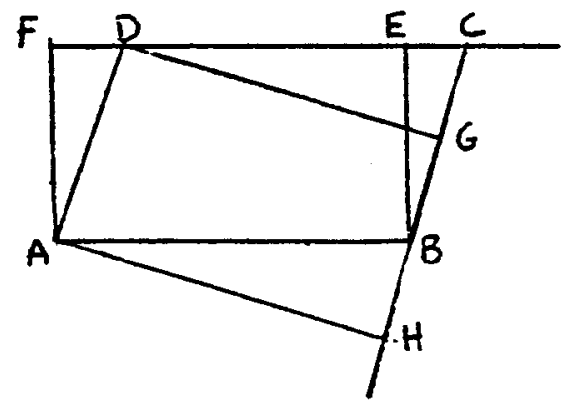

Comparer les aires des rectangles ABEF et $A D G H$.

Pour traiter ce problème, on a besoin de faire jouer un rôle symétrique aux côtés non parallèles du parallélogramme. Le problème ci-dessous peut préparer cette situation.

- On déforme un rectangle $A B C D$ en parallélogramme de deux manières:

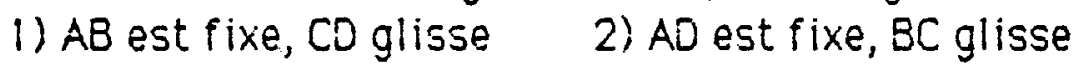

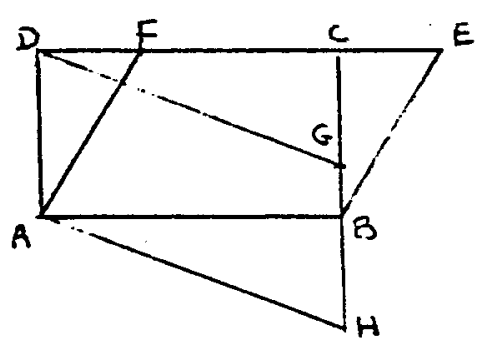

Comparer les aires des parallélogrammes ABEF et ADGH.

L'étude des problèmes de déformation ou de découpage et recollement font 
partie de la construction du sens de la formule usuelle $S=b \times h$. Mais ils ne peuvent pas ètre tous traités à l'école élémentaire. La déformation en parallèlogramme articulè peut être traitée en $C M$ jusqu'au cas limite d'aplatissement. En revanche, le glissement d'un côté sur son support peut être abordé seulement dans le cas ou il s'agit d'une autre manière de voir le découpage et recollement d'un triangle entièrement situé dans le parallèlogramme. Ces études suffisent déjà à dissocier les effets de ces deux déformations. C'est ce que nous avons observé lors des entretiens. Les autres cas et notamment le changement de base pourraient être abordés en CM2 mais aussi différés en Gème si_le temps à consacrer aux aires n'est pas suffisant à l'école élémentaire.

Sans l'étude des problèmes de changement de base, on s'appuie implicitement sur le fait qu'une surface a une aire bien déterminée et que des procédés différents de calcul doivent aboutir au même résultat. Or, J. Rogalski a observé que ce n'est pas un acquis pour tous les élèves de Sème dans le cas du triangle quelconque. La possibilité d'admettre l'unicité de la mesure quel. que soit le moyen de calcul, peut justifier qu'on introduise les formules (parallélogramme, triangle) à l'école primaire, vu lusage social qui en est fait. Toutefois, on voit le décalage qu'il y a entre la formule usuelle présentée dans toute sa genéralité "si simple", et le travail qu'il faudrait faire pour esperer asseoir sa validité. On ne peut pas s'étonner des difficultés et des dèrapages, par ailleurs couramment observés, chez des èlèves de collège, voire de lycee (nombreux encore sont ceux pour lesquels l'aire d'un parallélogramme s'obtient en multipliant les longueurs de deux cotés adjacents).

\section{Remarque}

Cette situation, proposee au CM2, a pour objectif de dissocier variation d'aire et variation de longueur des côtés d'une figure dans une déformation continue. A d'autres niveaux (4ème, 3ème), on pourrait aussi exploiter davantage la variation de longueur des diagonales.

\section{Jeu de cadres surfaces - aires - nombres}

C'est sur ces jeux de cadres ou' était basé notre premier processus d'apprentissage. Nous les reprenons et en résumons ci-dessous les enjeux.

A un moment de l'apprentissage ( $f$ in de $C M 1$ ou $C M 2$ ), on peut se trouver dans la situation suivante:

- aans le cadre géométrique, on sait comparer certaines surfaces par déplacement ou par découpage - recollement.

- dans le cadre numérique, on dispose des nombres entiers et de leurs opérations, on a commencé à étendre le domaine des nombres et on dispose de 
queloues nombres fractionnaires ou décimaux mais on n'a pas encore toutes les opérations sur ces nouveaux nombres.

- on sait associer un nombre à certaines surfaces par le comotage de carreaux sur papier quadrillé ; on a admis que le nombre de carreaux était invariant par déplacement de la surface et par découpage et recollement convenable.

L'extension de l'application mesure entre surfaces et nombres va permettre

1. d'étendre l'ensemble des surfaces dont on sait comparer les aires par pavage.

2. d'étendre la multiplication aux nombres fractionnaires et en particulier aux nombres décimaux.

Inversement, l'extension du champ des surfaces dont on sait comparer les aires par découpage-recollement et l'extension des operations sur les nombres permettent d'étendre l'application-mesure entre surfaces et nombres.

\section{a) pavage}

On a un premier moyen pour comparer davantage de surfaces : le pavage. On peut ainsi associer un nombre aux surfaces qu'on sait paver et comparer les nombres pour comparer les surfaces, à condition d'avoir utilisè des éléments de même aire (éventuellement de formes différentes) pour paver les surfaces à comparer. On constitue de la sorte des familles de surfaces comparables, mais il se peut qu'on ne sache pas comparer des surfaces appartenant à des familles diffèrentes.

Au cours du pavage, on peut rencontrer les situations suivantes:

- à une même surface, on a pu associer des nombres différents si elle a été pavée avec des éléments daires différentes.

- on a pavé des surfaces avec des surfaces élémentaires d'aires différentes. On ne peut ramener la comparaison des aires à celle des nombres par le pavage que si on a utilisé des éléments de même aire pour paver. On sera dans ce cas si on trouve une surface qui pave les différentes surfaces èlémentaires utilisèes.

Ces situations contribuent à dissocier l'aire d'une surface du nombre aui la' mesure et à mettre laccent sur le choix nécessaire d'une unite pour construire une application mesure, c'est pourouoi il nous parait important de les prendre en compte dans l'apprentissage.

A partir d'une activité géométrique, le pavage, et de ce quion sait sur les nombres, on étend les connaissances géométriques (davantage de surfaces comparables du point de vue de l'aire) et la relation entre surfaces et nombres (élaboration d'une notion d'application mesure).

Une unitè d'aire étant choisie, le pavage permet d'associer un nombre 
entier à certaines surfaces. Si on dispose d'autres nombres (petites fractions), on va pouvoir associer un nombre a davantage de surfaces en considérant des subdivisions d'un élément de pavage d'aire 1. Mais il reste des surfaces auxquelles on ne sait pas associer de nombre.

\section{b) extension de la multiplication}

Dans le jeu de cadres aires - surfaces - nombres, on va aussi récupérer de l'information nouvelle dans le cadre numérique : on peut donner du sens au produit des fractions et des décimaux en s'appuyant sur les aires de rectangles.

Supposons qu'on soit à un moment de l'apprentissage où

- une unité de longueur (resp. d'aire) étant choisie, on sait utiliser des fractions pour désigner des longueurs (resp. des aires, par mesure directe)

- pour des unités de longueur et d'aire adaptées, on sait que, pour un rectangle de dimensions entières, la mesure de laire est le produit des mesures des dimensions.

- on cherche à évaluer l'aire d'un rectangle de dimensions fractionnaires.

Par exemple $(3+4 / 5)$ et $(2+2 / 3)$

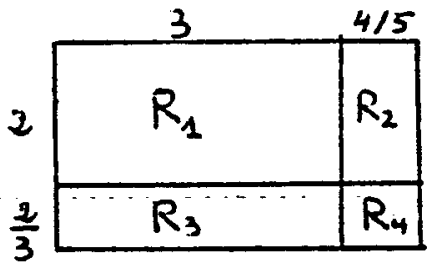

Fig 1

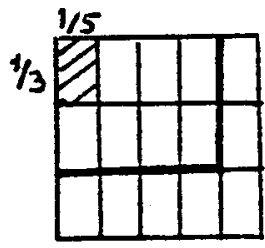

Fig 3

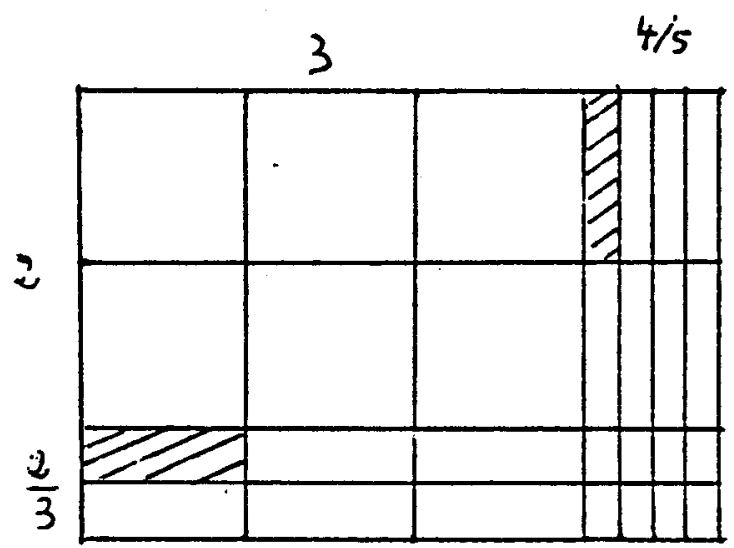

Fig 2

Le rectangle $R_{1}$ de dimensions $(3,2)$ a une aire qui mesure 6 car $2 \times 3=6:$ ii. contient 6 carrés unite ( $F$ ig 1 ).

Le rectangle de dimensions $(1 / 5,1)$ a une aire qui mesure $1 / 5$ car il se reporte 5 fois dans le carre unite, on en a $4 \times 2=8$ dans $R_{2}$ (Fig 2).

Le rectangle de dimensions $(1 / 3,1)$ a une aire qui mesure $1 / 3$ car il se reporte 3 fois dans le carré unité, on en a $\overline{5} \times 2=6$ dans $R_{3}$ (Fig 2).

Le rectangle $R_{4}$ de dimensions $(4 / 5,2 / 3)$ contient 8 petits rectangles de dimensions $(1 / 5,1 / 3)$; chacun de ces petits rectangles se reporte 15 fois dans le carré unité, et a donc une aire qui mesure $1 / 15$ (Fig 3). La mesure de 
Paire du rectangle $R_{4}$ est donc $8 / 15$. Ce résultat a été trouvé à partir des dimensions $4 / 5$ et $2 / 3$, du pavage et des règles d'écriture des fractions. On convient alors que $(4 / 5) \times(2 / 3)=8 / 15$.

On va ainsi donner comme sens au produit de deux nombres fractionnaires la mesure de l'aire d'un rectangle de dimensions ces deux nombres.

Le rectangle de dimensions $(3+4 / 5,2+2 / 3)$ a une aire qui mesure

$6+(8 \times 1 / 5)+(6 \times 1 / 3)+(8 \times 1 / 15)=9+3 / 5+8 / 15$.

Nous aurons donc $(3+4 / 5) \times(2+2 / 3)=(3 \times 2)+(3 \times 2 / 3)+(2 \times 4 / 5)+(4 / 5 \times 2 / 3)$

$=9+3 / 5+8 / 15$ et aussi $4 / 5 \times 2=8 / 5$ et $3 \times 2 / 3=6 / 3$.

Dans les deux derniers cas, ce sens de la multiplication coïncide avec celui de l'addition répétée qu'on avait pu rencontrer auparavant.

D'autres situations permettront de recoller les différents sens de la multiplication qu'on peut rencontrer.

Dans toute la suite du travail sur les aires, le jeu de cadres surfaces aires - nombres se poursuit, en particulier dans toutes les situations oui concernent les mesures:

- élaboration des formules de calcul daire des surfaces usuelles

- proportionnalité de la mesure de laire du rectangle à la mesure de chacune des dimensions

- bidimensionalité de l'aire: si on agrandit une surface dans un rapport $k$, son aire est multipliee par $k^{2}$.

Pour traiter chacun de ces problemes on s'appuie, par lintermediaire de divers découpages de surfaces, de pavage en même temps que d'addition et multiplication de nombres, sur l'interaction entre le cadre géométrique et le cadre numérique, l'aire étant l'invariant qui permet de relier les deux cadres.

\section{Conclusion}

Notre objectif était de construire la notion d'aire et une application mesure entre surfaces et nombres, de façon qu'elies prennent sens pour les éleves. Notre analyse a priori nous amenait à faire des choix didactiques dases sur des jeux de cadres surfaces-aires-nomores dans lesqueis les cadres differaient par leurs objets. Plus précisement, certaines séquences ont eu pour objet de rendre concevable la comparaison d'aires sans recours a la mesure. D'autres ont eu pour objet la comparaison et le calcul daires en recourant à la mesure dans le cas de suriaces pavabies avec des élements "étrangers" (non superposables ou ne provenant pas d'une subdivision de lun d'eux) et aussi dans le cas de surfaces non pavables avec un élement choisi à l'avance. Ce travail a permis aux éièves d'étendre le champ des surfaces qu'ils savaient mesurer avec une unité donnée, à des surfaces non pavables avec cette unité. En particulier, un acquis de leur apprentissage est qu'il est 
possible d'exprimer en $\mathrm{cm}^{2}$ l'aire d'un triangle, d'un parallèlogramme ou d'une autre surface, que le $\mathrm{cm}^{2}$ est l'aire d'une surface qui peut prendre des formes très diverses.

Autrement dit, une des conséquences de l'apprentissage est la prise de distance par rapport à la conception de l'aire liée à la forme.

Toutefois, cette évolution niest pas nécessairement un résultat qui intervient tout de suite après l'apprentissage. A ce moment là, au CMI, pour comparer des aires de surfaces usuelles, le decoupage et recollement convenable n'a été un outil opératoire que pour la moitié des élèves environ. Dans les deux classes, pour traiter ces questions certains amalgament encore aire et périmètre. D'autres les numérisent en recourant abusivement aux longueurs des côtés. D'autres enf in font intervenir fortement un point de vue "déformation" de surfaces usuelles (ici des parallélogrammes) en surfaces plus familières qu'ils savent traiter (des rectangles). La déformation dans le cas traité pouvait conserver laire (glissement dun côté du parallélogramme sur son support) ou ne pas la conserver (articulation de deux côtés autour des sommets). Elle produisait dans les deux cas une déclaration de conservation de l'aire. Les èlèves concernés ont fait évoluer leur point de vue de façon déterminante lors des entretiens en binôme, et ce de façon d'autant plus marquee que leurs conceptions les conduisaient à des affirmations contradictoires.

Ainsi, l'expérience nous a appris que les jeux de cadres prèvus étaient insuffisants et qu'il etait important d'y ajouter des jeux entre cadres qui diffèrent par la problématique - ici statique, dynamique - comme nous l'avons décrit ci-dessus. Les situations à proposer aux élèves restent à expérimenter et à mettre au point.

Nous pensons quaprès ce travail, on devrait être mieux armé pour identifier aires et nombres avec un moindre risque de dérapage.

on pourrait même atteindre les aires de surfaces pour lesquelles le maillage carré n'est pas nécessairement le meilleur et pour lesquelles la longueur du bord n'est pas nécessairement finie. Par exemple S, limite de la suite $S_{n}$ :

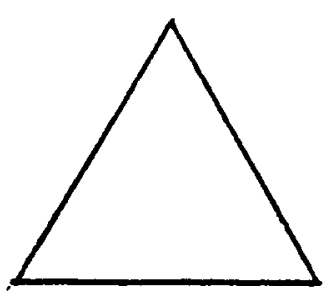

$S_{0}$

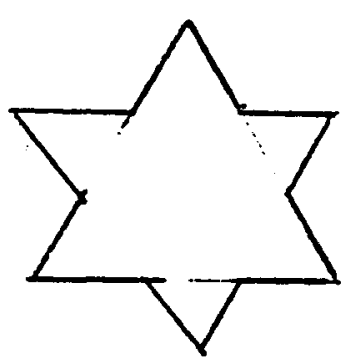

$S_{1}$

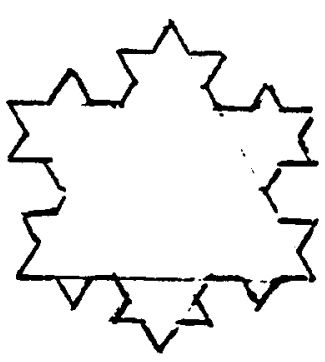

$\mathrm{S}_{2}$ 
On fabrique $S_{n+1}$ à partir de $S_{a}$ en ajoutant un triangle équilatéral construit sur le tiers central de chaque côté.

\section{Références:}

R. DOUADY M.J. PERRIN Aires de surfaces planes lère partie et 2ème partie "Petit $x " n$ " $\sigma($ dec. 84$)$ et "petit $x^{\prime \prime} n " g($ juin 85) IREM de Grenoble.

R. DOUADY M.J. PERRIN Mesure des longueurs et des aires. Brochure $n^{\circ} 48$ (sept. 1983) IREM Université Paris 7.

J. ROGALSKI L'acquisition des notions relatives à la dimensionalité des mesures spatiales (longueur; surface).-Recherches en Didactique-des mathematiques n'3.31983 La Pensée Sauvage

VINH BANG et LUNZER. E. Conservations spatiales Etudes d'épistémologie genétique vol 19 Paris PUF 1965 
Combien de petites figures dans be grande? (entoure la borne réponse)

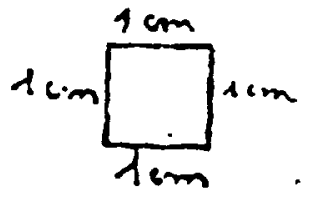

(corré)
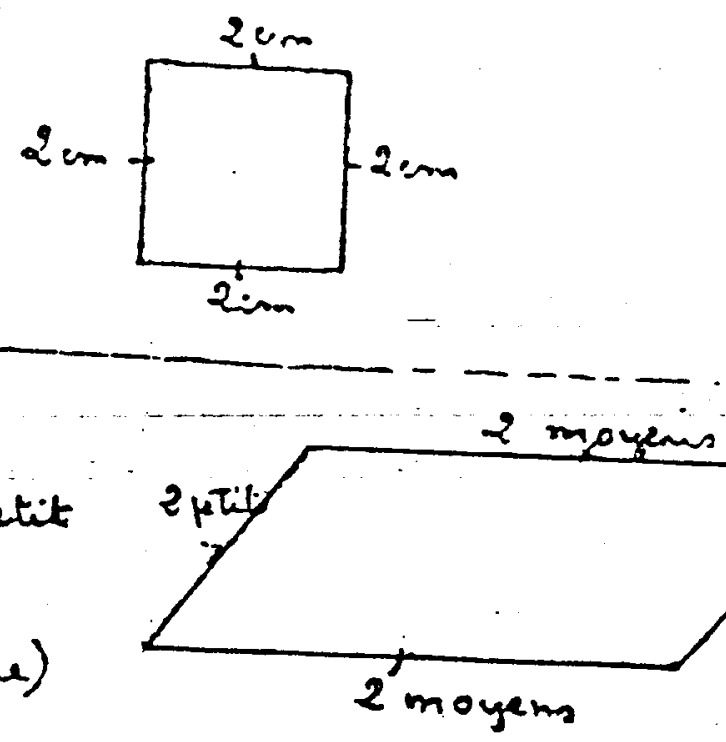

(sorablélogranme)
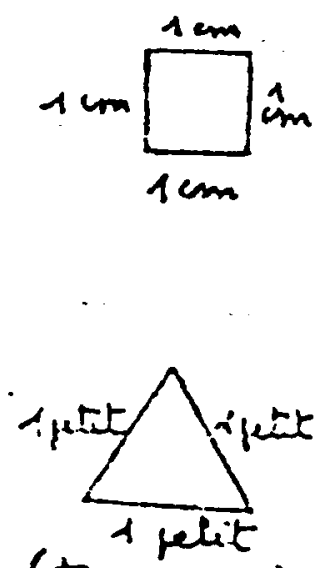

(triangle)
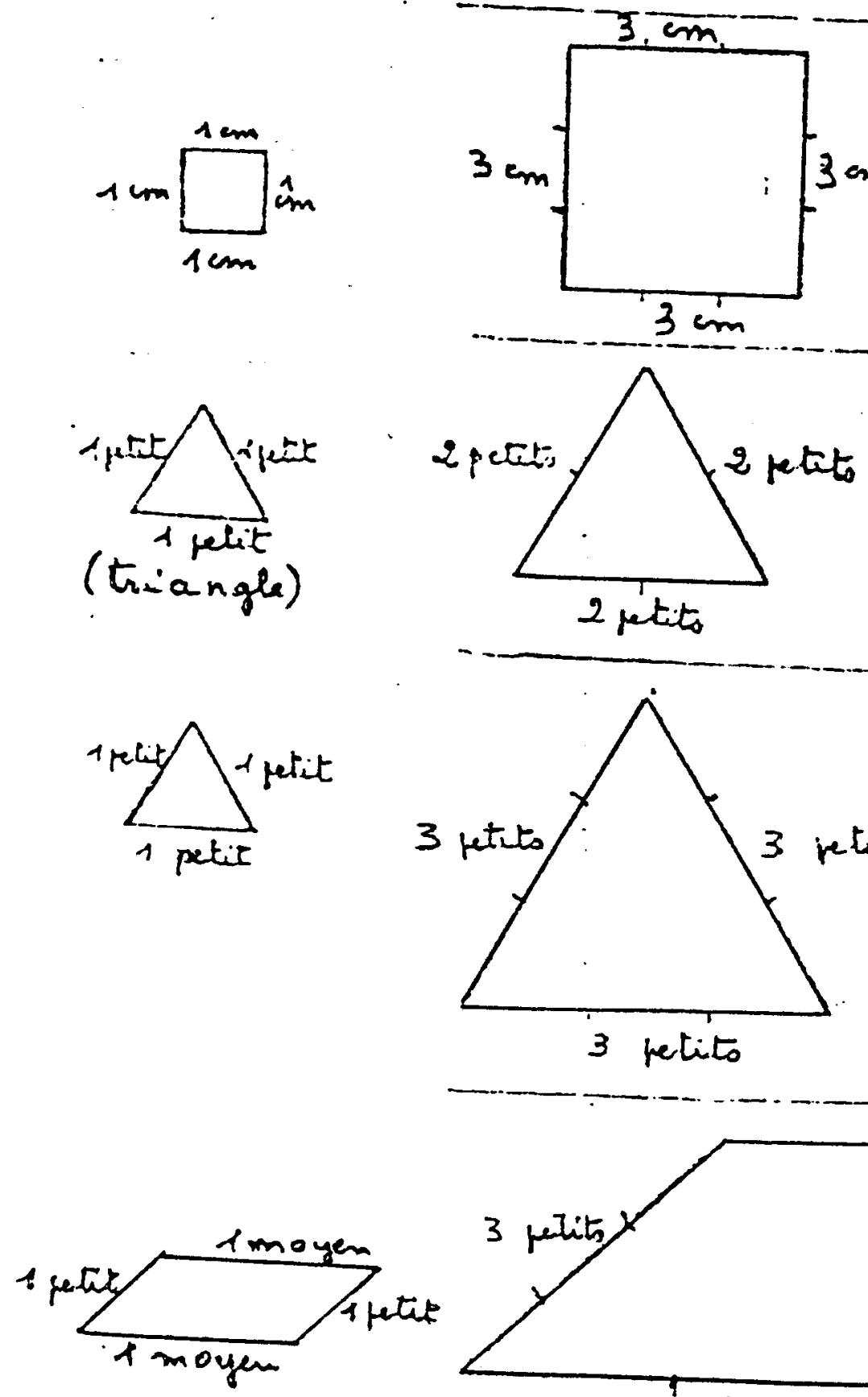

3 retito
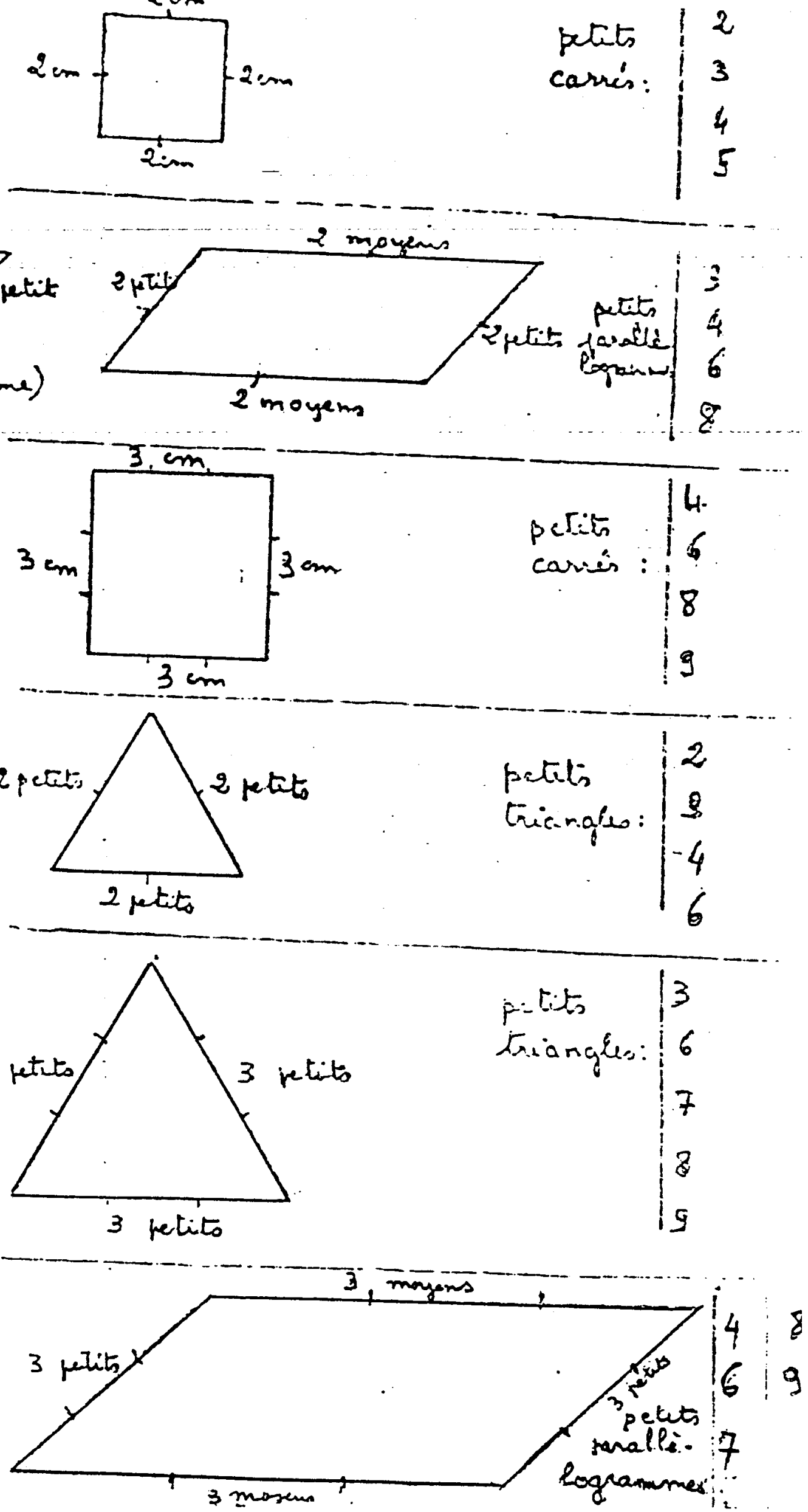
Poser les opérations effectuées.

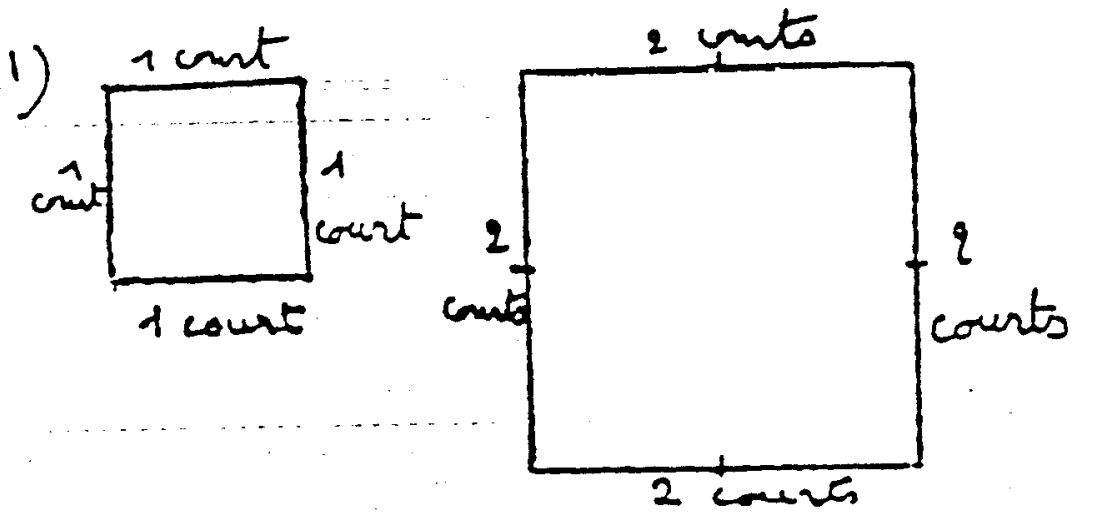

Pour peindre le petit cerre', it fout ? 5 purts 5 ots de peinture raxe contrien de prto powa le grand?

2) 1 manen

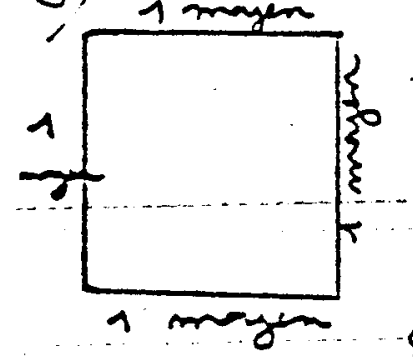

3 myens

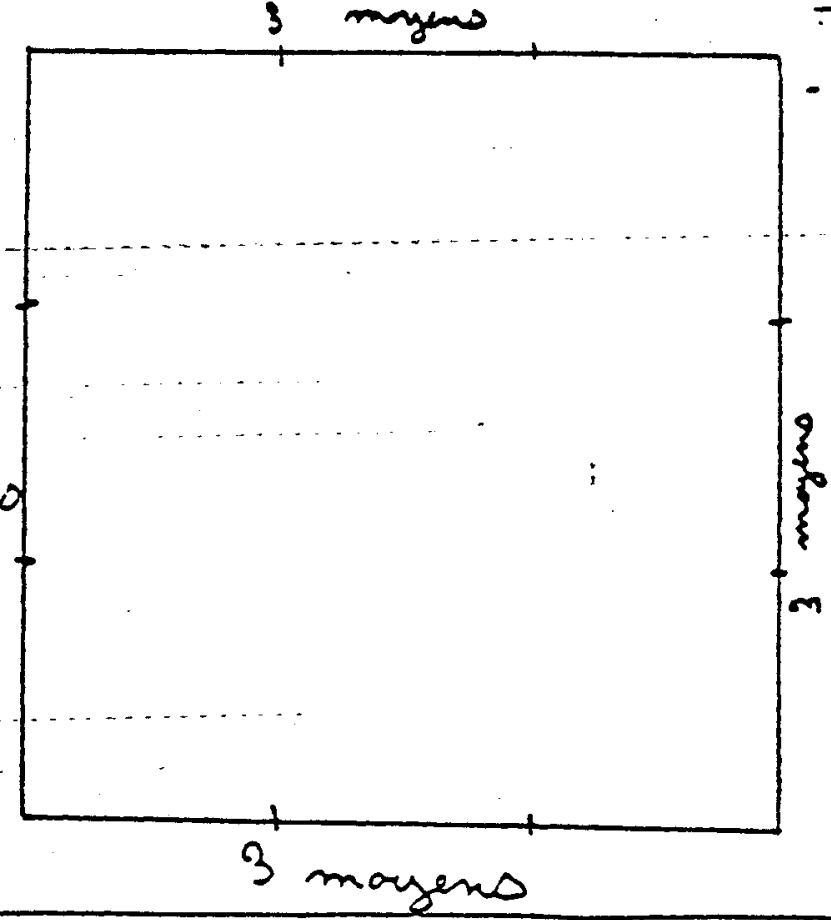

- Pour peindre le petit carré, il taut 6 pots de peintire verte. Combien de pots pour be grand?

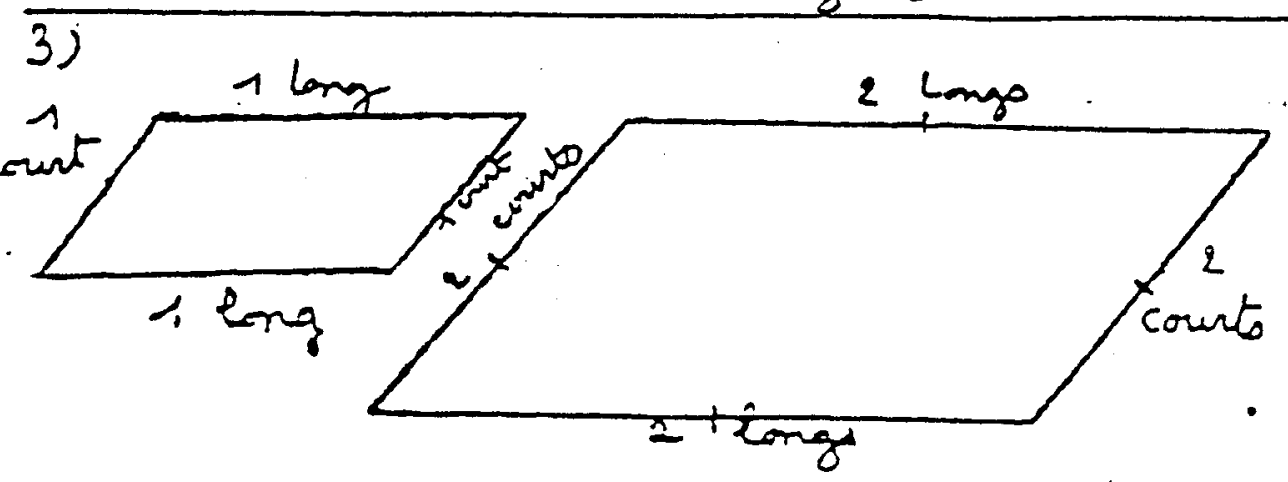

Pour puináre le pectit parallélogramm, il fait Lits 5 pots de peinters jocence Combien de pats par li grend?

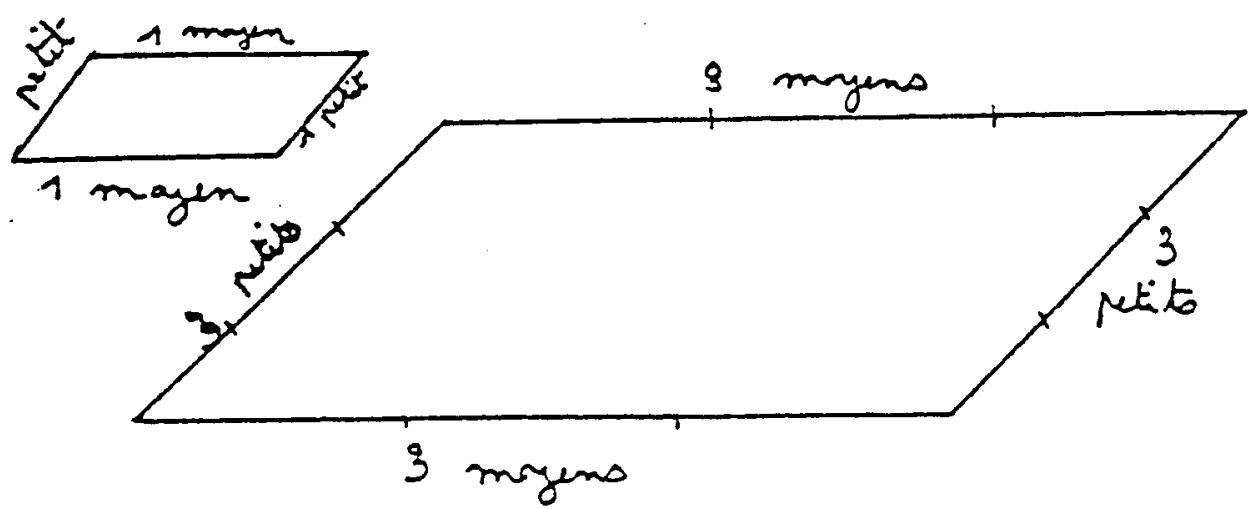

Pour peindre ke petit parallibgremene, ilfoict 4 poto de peinture bleus. combien de pots pank gavex. 
Poser les operations effectisés.

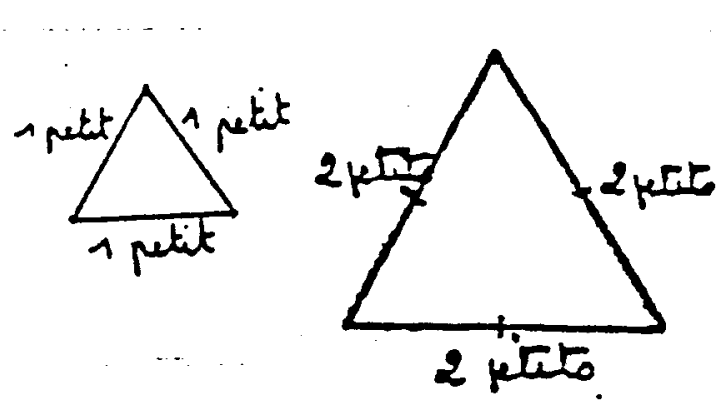

Pour peindre le petit triangle, il paut a pot de peinture blenche. combiende poto pou le grand?
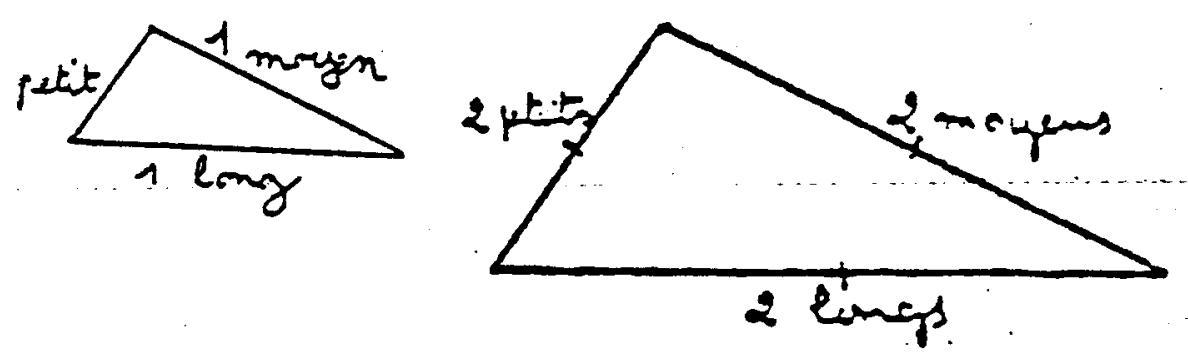

Pour peindre le potit triorfis. il fant 2 poto de peenture marron.

Combien de poto pour le grand!
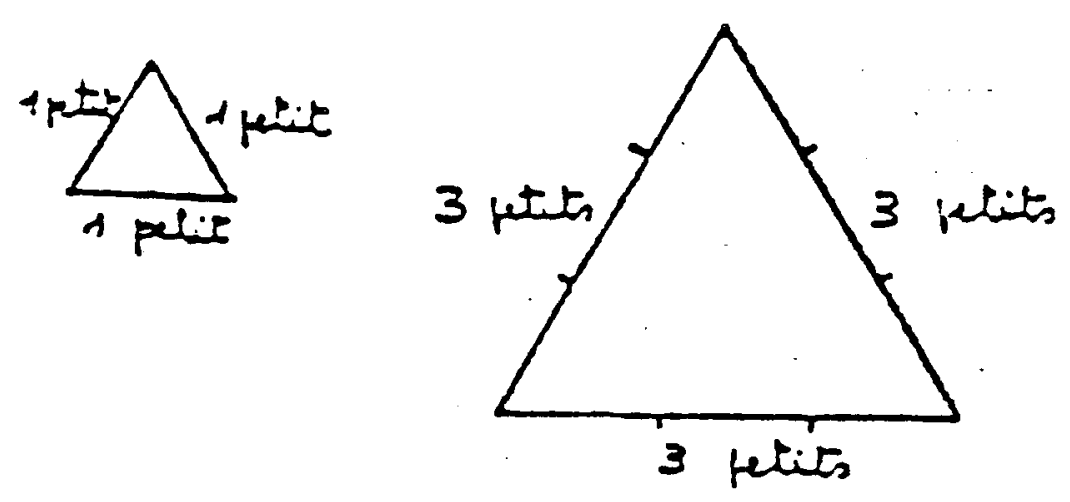

Pour peindre lis pectit triangli, il paut 1 pot de peintur. olange.

Combien de pots pour le-gread?

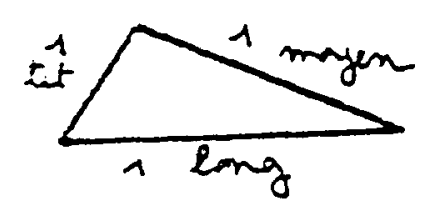

Pour peimalre le petit trianghe, it faut 2 pots de paintere grise. Combien de poto pros le giend?

is longs 
Poser les apeirations effecticés

la suiface des petito figures est hachuré . et voici leur mesure
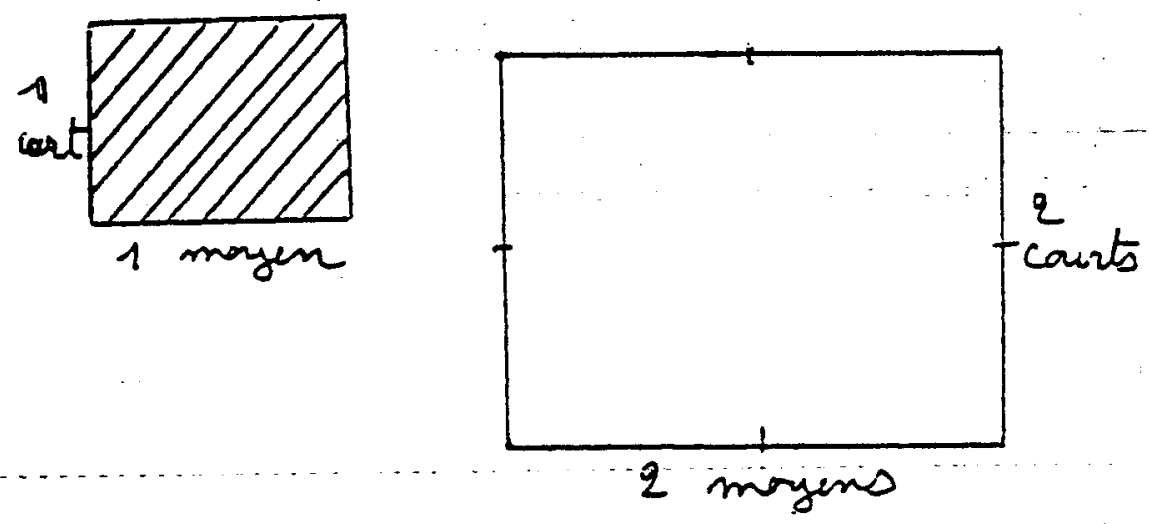

La surface du petit rectangle mesure $5 \mathrm{~cm}^{q}$. Combien meare la suppece due grand?

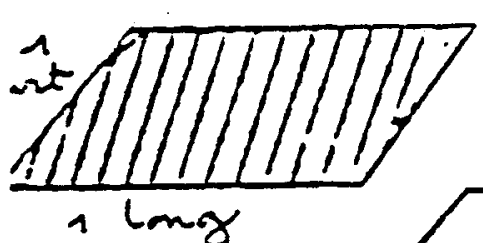

La surfuce du petit parallélaysemme mexure $5 \mathrm{~cm}^{2}$.

Combien mesure la surface du grand?

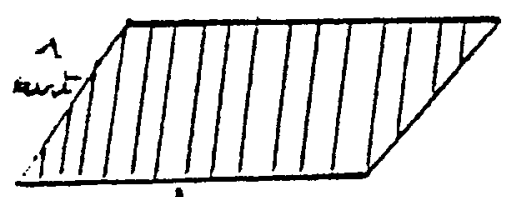

1 lang

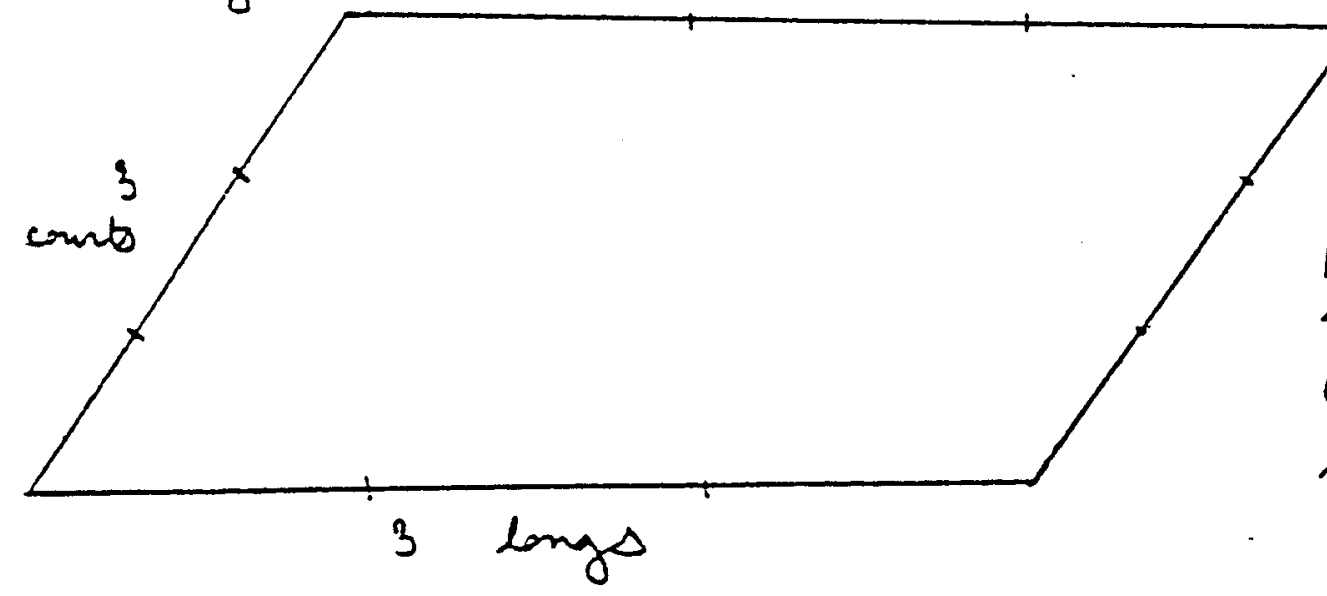

la supface du petit parullibogremone mexure $5 \mathrm{~cm}^{?}$. Combin mesuse ta supfice due grand? 
Poser Les opeirations effectués.

La sunface des petites figures est hachursé at voici leur mesuex
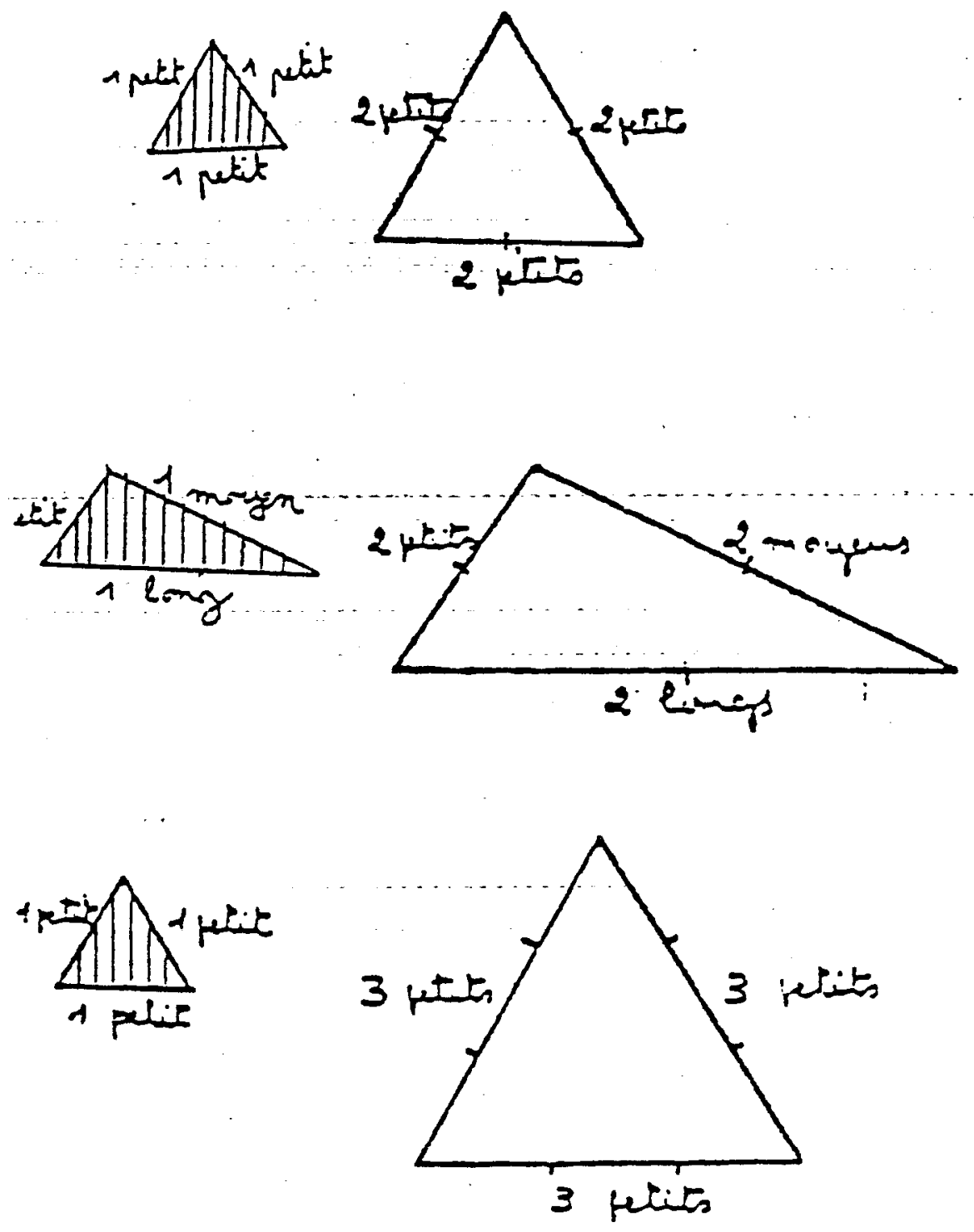

La surface du petit triangle mase $1 \mathrm{~cm}^{2}$. Combien masue la surface du grend?

La. surpace du pett triangh meare $2 \mathrm{~cm}^{?}$ ? Contrien mesure ba surfece due greand?

La surfece du potit triangle mexine $1 \mathrm{~cm}^{2}$. cantrien meare ha sufface due graind?

La sufface du petit triangle mesure $i \mathrm{~cm} q$. Combien mesure da Aunface du grand

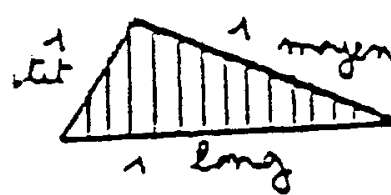

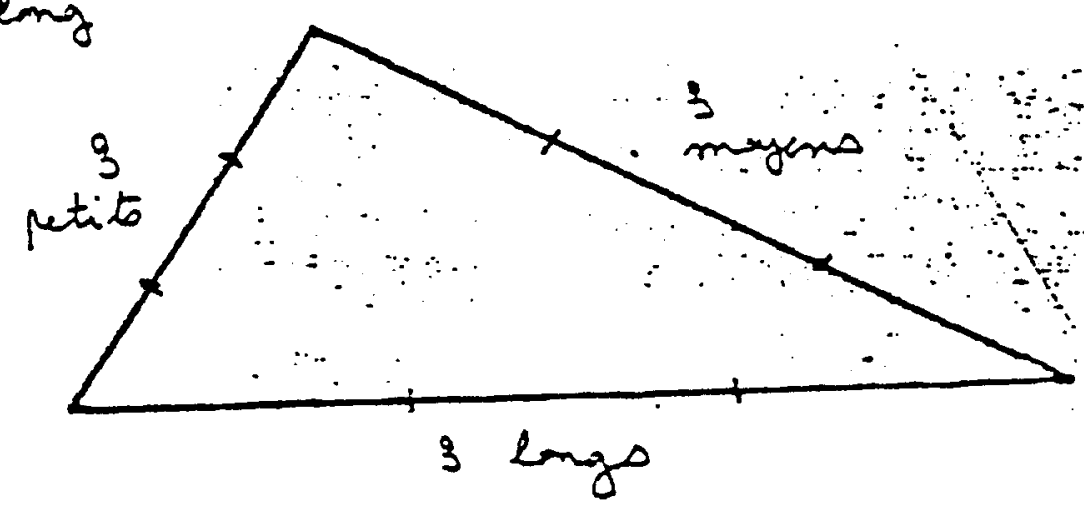




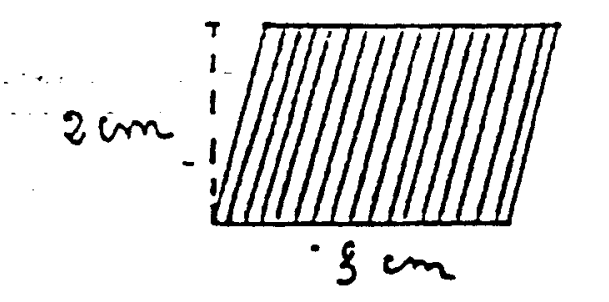

Dissine un rictangle de même aire
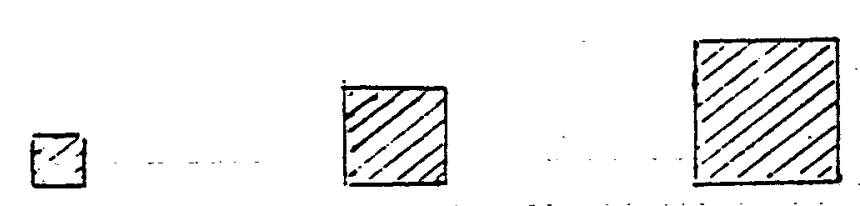

qual est le carré. qui a une aire de $1 \mathrm{~cm}^{2}$ ?

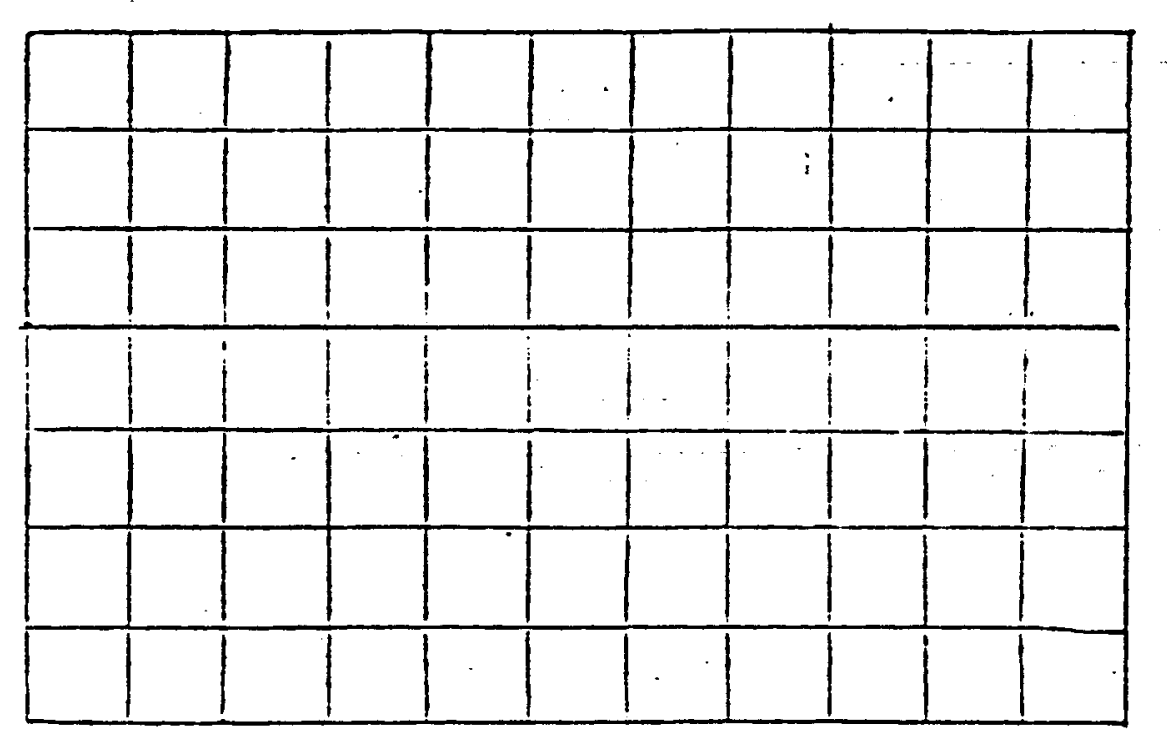

Cimbirin de carreaux dero:

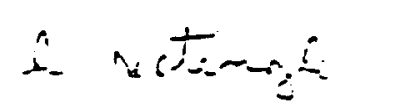

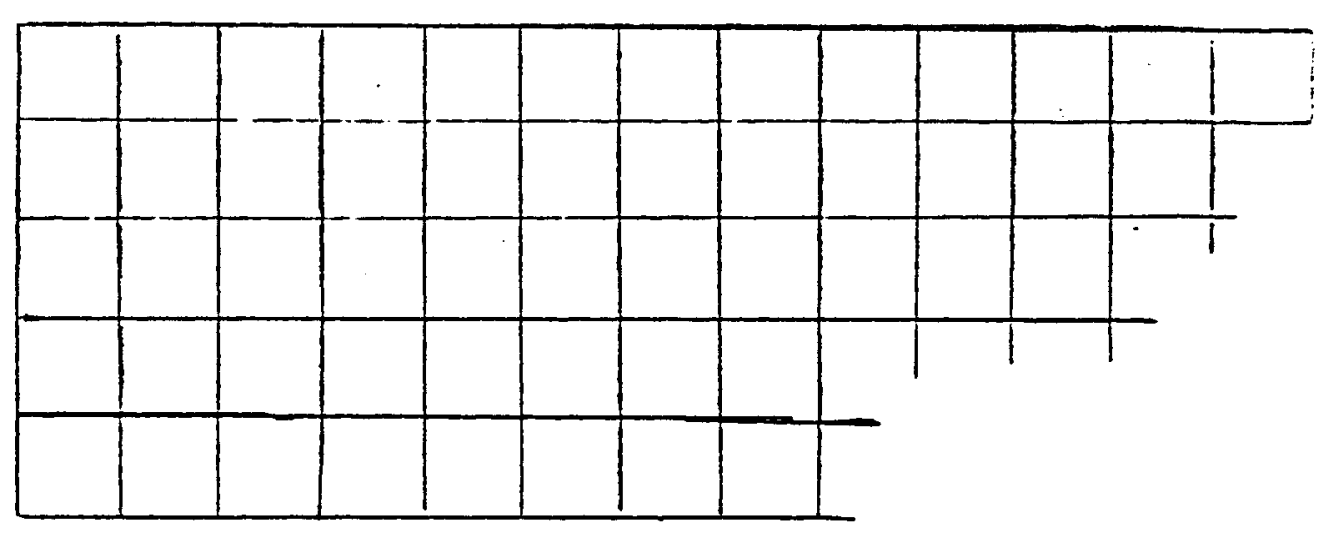

En a dichiré un morceau. Combien de corrsux y avait.il dans $k$ rectangle, borsqu'il itact entier? 


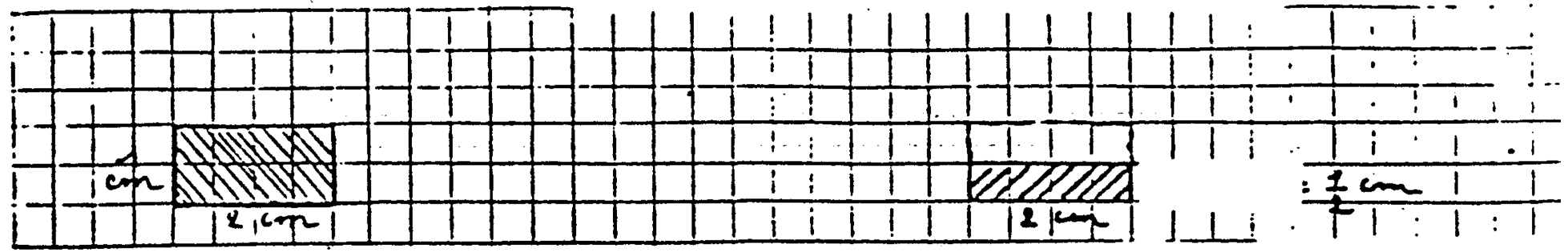

L'acie de ce rectangle est $2 \mathrm{~cm}^{2}$

Comfien moure la
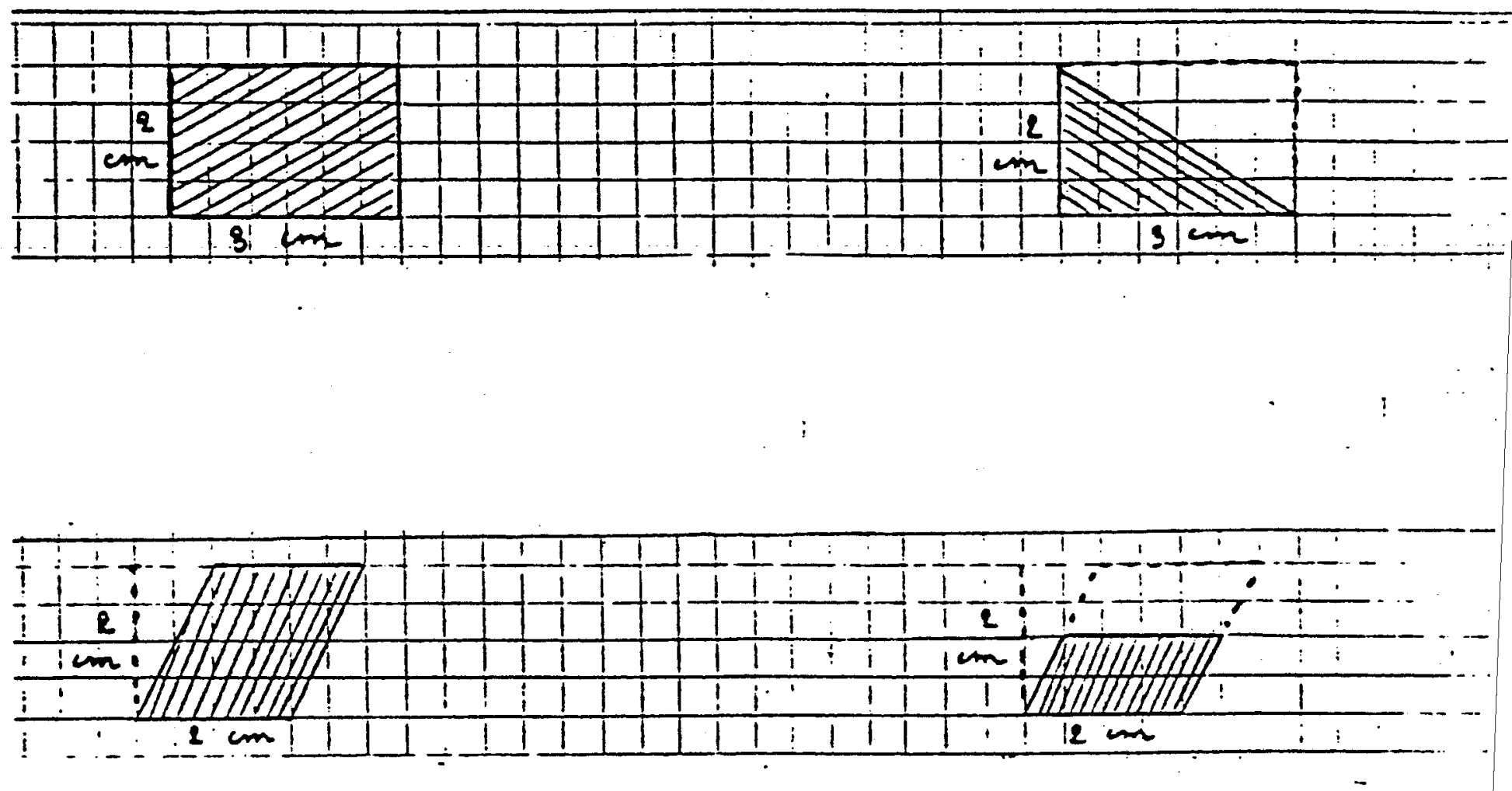

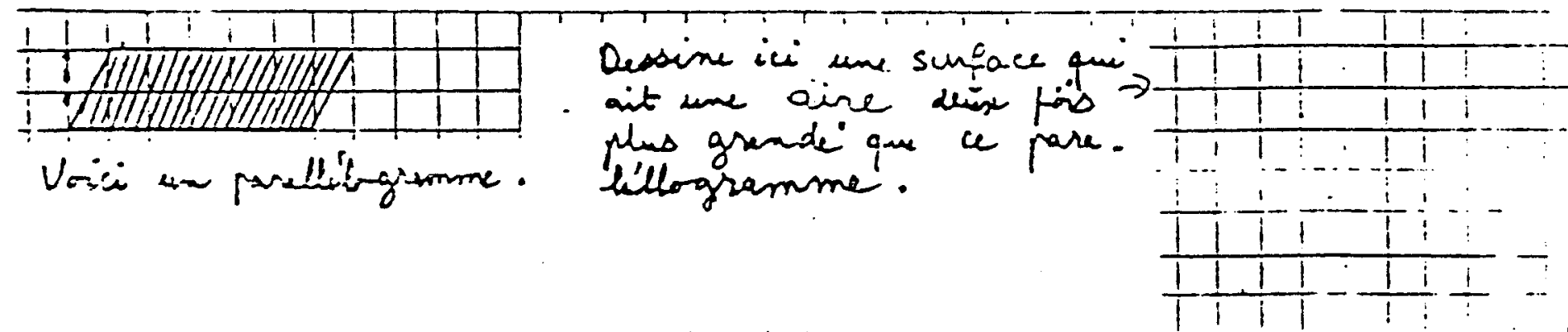

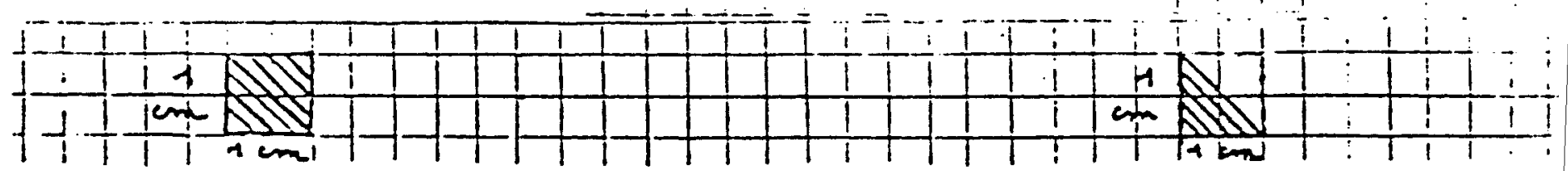

